\title{
Single-particle and collective excitations in quantum wires made up of vertically stacked quantum dots: Zero magnetic field
}

\author{
Manvir S. Kushwaha \\ Department of Physics and Astronomy, Rice University, P.O. Box 1892, Houston, TX 77251, USA
}

(Dated: March 4, 2018)

\begin{abstract}
We report on the theoretical investigation of the elementary electronic excitations in a quantum wires made up of vertically stacked self-assembled InAs/GaAs quantum dots. The length scales (of a few nanometers) involved in the experimental setups prompt us to consider an infinitely periodic system of two-dimensionally confined (InAs) quantum dot layers separated by GaAs spacers. The resultant quantum wire is characterized by a two-dimensional harmonic confining potential in the $x-y$ plane and a periodic (Kronig-Penney) potential along the $\mathrm{z}$ (or the growth) direction within the tight-binding approximation. Since the wells and barriers are formed from two different materials, we employ the Bastard's boundary conditions in order to determine the eigenfunctions along the z direction. These wave functions are then used to generate the Wannier functions, which, in turn, constitute the legitimate Bloch functions that govern the electron dynamics along the direction of periodicity. Thus the Bloch functions and the Hermite functions together characterize the whole system. We then make use of the Bohm-Pines' (full) random-phase approximation in order to derive a general nonlocal, dynamic dielectric function. Thus developed theoretical framework is then specified to work within a (lowest miniband and) two-subband model that enables us to scrutinize the single-particle as well as collective responses of the system. We compute and discuss the behavior of the eigenfunctions, band-widths, density of states, Fermi energy, single-particle and collective excitations, and finally size up the importance of studying the inverse dielectric function in relation with the quantum transport phenomena. It is remarkable to notice how the variation in the barrier- and well-widths can allow us to tailor the excitation spectrum in the desired energy range. Given the advantage of the vertically stacked quantum dots over the planar ones and the foreseen applications in the single-electron devices and in the quantum computation, it is quite interesting and important to explore the electronic, optical, and transport phenomena in such systems.
\end{abstract}

PACS numbers: 73.21.-b, 73.22.-f, 73.63.-b, 78.67.-n 


\section{INTRODUCTION}

Even though the foundation of lower-dimensional electron systems was laid much before (than thought), the discovery of quantum Hall effects (both integral and fractional) is known to have spurred the tremendous efforts to see the consequent changes with the reduction in the system's dimensions from three to two, two to one, and one to zero. These quasi- $n$-dimensional semiconductor heterostructures - with $n(=2,1$, or 0$)$ being the degree of freedom - are also known as quantum wells, quantum wires, and quantum dots in which the charge carriers exposed to external probes such as electric and/or magnetic fields can exhibit unprecedented quantal effects that strongly modify their behavior. Thanks to the advancements in the nanofabrication technology and electron lithography, these man-made quantum structures have paved the way to much of the exotic (fundamental and applied) physics emerged during the past two decades [1].

Years before vertically stacked self-assembled quantum dot molecules were synthesized, Sakaki had made a very precise diagnosis of the relevant parameters defining the quantum transport in coupled quantum dot arrays in the quantum limit [2]. This led him to propose the designing of such heterostructures with the optical phonon scattering practically eliminated. It was argued that the optical phonon scattering could be completely ruled out provided that the miniband (minigap) width is small (large) enough as compared to the optical phonon energy. It is generally recognized that the absence of phonon scattering leads to dramatic effects in the transport phenomena. For instance, it causes the efficient acceleration of electrons, which clearly improves the chances of the Bloch oscillations, so severely limited in the conventional systems.

The first vertically stacked self-assembled quantum dots were observed in InAs islands separated by GaAs spacer layers along the growth direction in 1995 [3]. What followed is a long list of experimental [4-23] and theoretical [24-40] works dealing with the elastic, electrical, electronic, and optical phenomena which led the researchers to visualize a variety of solid-state devices [5]. It is already well-established that the strain due to the lattice mismatch at the interfaces between two (different) semiconductors is the driving force for the growth of the self-assembled quantum dots and is becoming known to play a crucial role in determining diverse electronic and optical properties of the final system of vertically stacked quantum dots (VSQD).

The feasibility of varying the coupling strength (of the molecular bond) at will not only leads to some significant applications in spintronics [15], quantum computation [21], solar cells [36], and quantum optics [39] but also creates a fertile ground for exploring new fundamental physics such as manipulating the spins [15] and electrical control of electron g factors [37]. The most important 
and interesting (geometrical) aspect associated with the vertically stacked quantum dot system is that it has provided, for the first time, a reasonable venue for reversing the trend that began with the quest of diminishing dimensions in the mid 1980s. In other words, a VSQD structure with a strong coupling along the growth direction offers a quasi-one-dimensional (Q1D) system made up of quasi-zero-dimensional systems and hence the term "reversing the trend".

Literature is a live witness that the plasmon, in classical as well as quantum systems, has always drawn more attention than any other quasi-particle due, in fact, to its fundamental importance to the understanding of the electronic, optical, and transport phenomena in condensed matter physics [1]. And yet, it is really surprising that except for Ref. 34 no experimental and/or theoretical work on VSQD has, to the best of our knowledge, touched upon the subject. The present paper helps fill that void. Here, we embark on a systematic route to the investigation of the single-particle and collective excitations in the quantum wire made up of VSQD within a two-subband model in the framework of Bohm-Pines' random-phase approximation (RPA) [41] in the absence of an applied magnetic field.

It is noteworthy that the theoretical development in quantum wires has long suffered from an intense controversy over whether the system is best describable as Tomonaga-Luttinger liquid or as Fermi liquid. This issue was elegantly resolved by Das Sarma and coworkers [42] who rigorously justified the use of Fermi-liquid-like theories (such as the RPA) for describing the realistic quantum wires. It should be pointed out that our theoretical framework differs crucially from Ref. 34 where the authors followed the effective-mass approximation [see Sec. II for details]. For the extensive review on the plasmons in quantum wells, wires, dots, and their periodic counterparts, the reader is referred to Ref. 1.

The rest of the article is organized as follows. In Sec. II, we present the theoretical framework leading to the derivation of nonlocal, dynamic, dielectric function, which is further diagnosed analytically to fully address the solution of the problem and the related relevant aspects. In Sec. III, we discuss several illustrative examples of, for example, excitation spectrum comprising of single-particle and collective excitations, the influence of the variation of well- and barrierthicknesses, and highlight the importance of studying the inverse dielectric function in relation with the transport phenomena [43] in such quantum systems. Finally, we conclude our finding with specific remarks regarding the interesting features worth adding to the problem in Sec. IV. 


\section{THEORETICAL FRAMEWORK}

\section{A. Eigenfunctions and eigenenergies}

We consider a periodic system of quasi-two-dimensional InAs islands of thickness $a$ separated by GaAs spacer layers of thickness $b$. Each of the InAs island is constrained by a two-dimensional harmonic confining potential of the form of $V(x)=\frac{1}{2} m^{*} \omega_{0}^{2}\left(x^{2}+y^{2}\right)$ in the $\mathrm{x}-\mathrm{y}$ plane. The $\mathrm{z}$ (i.e., the growth) direction is assumed to be under the greater confinement potential, say, $V_{c}(z)$, that allows strong coupling between the InAs islands. The small length scales and strong coupling cause the resultant structure (see Fig. 1) to mimic a realistic quantum wire with a practically well-defined linear charge density $\left(n_{1 D}\right)$. Since the coupling strength and hence the tunneling can be controlled by varying the thickness of the barrier between the quantum dot layers, we believe that the use of the (traditional) tight-binding approximation (TBA) is quite justifiable [33]. Next, we consider the resultant system with moderate tunneling (in the polarizability function) describable with the energy dispersion due to tunneling being sinusoidal. Such a system as described above can be formally characterized by the eigenfunction
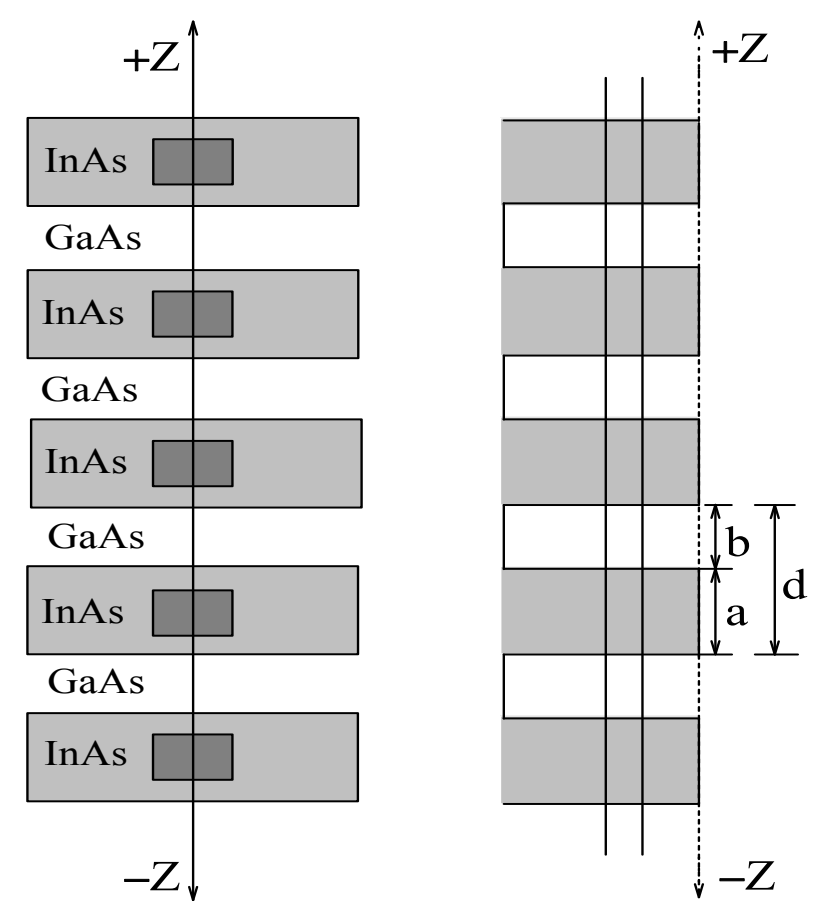

FIG. 1: Schematic of the quantum wire made up of an infinitely periodic system of InAs islands separated by GaAs spacer layers (left panel). The right panel shows the Kronig-Penney periodic-potential simulation along the growth direction. Here $a(b)$ is the well (barrier) width and $d=a+b$ is the period of the resultant system making up quantum wire. 


$$
\psi(x, y, z)=\phi_{n_{x}}(x) \phi_{n_{x}}(y) \phi_{t}(z)
$$

where

$$
\phi_{n_{s}}(s)=N_{n_{s}} e^{-s^{2} / 2 \ell_{c}^{2}} H_{n_{s}}\left(s / \ell_{c}\right),
$$

where $s \equiv x, y, \ell_{c}=\sqrt{\hbar / m^{*} \omega_{0}}$ is the characteristic length, $\omega_{0}$ is the frequency of the harmonic oscillator, $N_{n}=1 / \sqrt{\sqrt{\pi} 2^{n} n ! \ell_{c}}$ is the standard normalization constant, and $H_{n_{s}}\left(s / \ell_{c}\right)$ is the Hermite polynomial, and

$$
\phi_{t}(z)=\frac{1}{\sqrt{N}} \sum_{l} e^{i k l d} \chi_{t}(z-l d),
$$

where $d=a+b$ is the period, $k$ the Bloch vector, $\chi_{t}(\ldots)$ the well-known Wannier function, and the eigenenergy

$$
\epsilon_{k n t}=(n+1) \hbar \omega_{0}+\epsilon_{t}-\frac{W_{t}}{2} \cos (k d)
$$

where $t\left(n=n_{x}+n_{y}\right)$ is the miniband (subband) index, $\epsilon_{t}$ the energy of the $t$-th miniband and $W_{t}$ is the band-width defined as

$$
W_{t}=-4 \int_{-a / 2}^{+a / 2} d z \chi_{t}(z) V_{0} \chi_{t}(z-d),
$$

where we assume that the confining potential is a finite square well (Kronig-Penney potential) with a barrier height $V_{0}$ and well-width $a$. Since $N$ (the number of quantum dot layers) is very large, the sum in Eq. (3) can be written as integral according to the replacement rule: $\sum_{k} \rightarrow$ $\left(N / L_{z}\right) \int_{B Z} d k$. Note that Eq. (3) represents the tight-binding constraint which hypothesizes a little overlap between the wave functions of different sites. There $\chi_{t}(\ldots)$, if normalized in the length of the lattice (or, nearly enough, in infinite length), satisfies: $\int d z \chi_{t}^{*}(z-n d) \chi_{t}(z-l d)=\delta_{n l}$ and $\int d z \phi_{t}^{*}(z) \phi_{t}(z)=1$. Here $L_{z}=N d$ is the total crystal length along the growth direction.

\section{B. Nonlocal, dynamic dielectric function}

We start with the general expression of the non-interacting single-particle density-density response function $(\mathrm{DDRF}) \chi^{0}(\ldots)$ given by $[1]$ 


$$
\chi^{0}\left(\mathbf{r}, \mathbf{r}^{\prime} ; \omega\right)=\sum_{i j} \Lambda_{i j} \psi_{i}^{*}(\mathbf{r}) \psi_{j}(\mathbf{r}) \psi_{j}^{*}\left(\mathbf{r}^{\prime}\right) \psi_{i}\left(\mathbf{r}^{\prime}\right)
$$

where $\mathbf{r} \equiv(x, y, z)$, the composite index $i, j \equiv k, n, t$, and $\Lambda_{i j}$ is defined as follows.

$$
\Lambda_{i j}=2 \frac{f\left(\epsilon_{i}\right)-f\left(\epsilon_{j}\right)}{\epsilon_{i}-\epsilon_{j}+\hbar \omega^{*}}
$$

where $f(x)$ is the well-known Fermi distribution function. $\omega^{*}=\omega+i \gamma$ and small but nonzero $\gamma$ represents the adiabatic switching of the Coulomb interactions in the remote past. The factor of 2 takes care of the spin degeneracy.

Next, we write the induced particle density with the aid of the Kubo's correlation function [1]. The result is

$$
\begin{aligned}
n_{\text {in }}(x, y, z ; \omega) & =\int d x^{\prime} \int d y^{\prime} \int d z^{\prime} \chi^{0}\left(x, y, z ; x^{\prime}, y^{\prime}, z^{\prime} ; \omega\right) V_{t o t}\left(x^{\prime}, y^{\prime}, z^{\prime} ; \omega\right) \\
& =\int d x^{\prime} \int d y^{\prime} \int d z^{\prime} \chi\left(x, y, z ; x^{\prime}, y^{\prime}, z^{\prime} ; \omega\right) V_{e x}\left(x^{\prime}, y^{\prime}, z^{\prime} ; \omega\right),
\end{aligned}
$$

where $V_{t o t}=V_{e x}+V_{i n}$ is the total potential, with $V_{e x}\left(V_{i n}\right)$ as the external (induced) potential. $\chi$ and $\chi^{0}$ are, respectively, the total and the single-particle DDRF and are related to each other through an integral Dyson equation

$$
\chi\left(\mathbf{r}, \mathbf{r}^{\prime} ; \omega\right)=\chi^{0}\left(\mathbf{r}, \mathbf{r}^{\prime} ; \omega\right)+\int d \mathbf{r}^{\prime \prime} \int d \mathbf{r}^{\prime \prime \prime} \chi^{0}\left(\mathbf{r}, \mathbf{r}^{\prime \prime} ; \omega\right) V_{e e}\left(\mathbf{r}^{\prime \prime}, \mathbf{r}^{\prime \prime \prime}\right) \chi\left(\mathbf{r}^{\prime \prime \prime}, \mathbf{r}^{\prime} ; \omega\right),
$$

where $V_{e e}(\ldots)$ represents the binary Coulomb interactions and is defined as

$$
V_{e e}\left(\mathbf{r}, \mathbf{r}^{\prime}\right)=\frac{e^{2}}{\epsilon_{b}} \frac{1}{\left|\left(x-x^{\prime}\right)^{2}+\left(y-y^{\prime}\right)^{2}+\left(z-z^{\prime}\right)^{2}\right|^{1 / 2}},
$$

where $-e(e>0)$ is the elementary charge and $\epsilon_{b}$ the background dielectric constant of the system. Further, the induced potential in terms of the induced particle density is expressed as

$$
V_{i n}(x, y, z ; \omega)=\int d x^{\prime} \int d y^{\prime} \int d z^{\prime} V_{e e}\left(x-x^{\prime}, y-y^{\prime}, z-z^{\prime}\right) n_{i n}\left(x^{\prime}, y^{\prime}, z^{\prime} ; \omega\right)
$$

Equation (11), with the aid of Eqs. (1), (3), (6), (8), and (10), yields 


$$
\begin{aligned}
V_{i n}(x, y, z ; \omega)= & \frac{e^{2}}{\epsilon_{b}} \frac{1}{N^{2}} \sum_{k k^{\prime}} \sum_{n n^{\prime}} \sum_{t t^{\prime}} \sum_{l l^{\prime}} \Lambda_{n n^{\prime}}\left(k, k^{\prime} ; \omega\right) e^{i q l d} e^{-i q l^{\prime} d} \\
& \times \int d x^{\prime} \int d y^{\prime} \int d z^{\prime} \int d x^{\prime \prime} \int d y^{\prime \prime} \int d z^{\prime \prime} \\
& \times \frac{1}{\left|\left(x-x^{\prime}\right)^{2}+\left(y-y^{\prime}\right)^{2}+\left(z-z^{\prime}\right)^{2}\right|^{1 / 2}} \\
& \times \phi_{n_{x}}^{*}\left(x^{\prime}\right) \phi_{n_{y}}^{*}\left(y^{\prime}\right) \phi_{n_{x}^{\prime}}\left(x^{\prime}\right) \phi_{n_{y}^{\prime}}\left(y^{\prime}\right) \phi_{n_{x}^{\prime}}^{*}\left(x^{\prime \prime}\right) \phi_{n_{y}^{\prime}}^{*}\left(y^{\prime \prime}\right) \phi_{n_{x}}\left(x^{\prime \prime}\right) \phi_{n_{y}}\left(y^{\prime \prime}\right) \\
& \times \chi_{t}^{*}\left(z^{\prime}-l d\right) \chi_{t^{\prime}}\left(z^{\prime}-l d\right) \chi_{t^{\prime}}^{*}\left(z^{\prime \prime}-l^{\prime} d\right) \chi_{t}\left(z^{\prime \prime}-l^{\prime} d\right) \\
& \times V_{t o t}\left(x^{\prime \prime}, y^{\prime \prime}, z^{\prime \prime} ; \omega\right),
\end{aligned}
$$

where $k^{\prime}=k+q$ and $q$ is the momentum transfer. Next, we follow two steps: (i) multiply both sides of Eq. (12) by $e^{-i q^{\prime} z}$ and integrate with respect to z, and (ii) introduce, for convenience, a single-Fourier component of the total potential to write $V_{t o t}\left(x^{\prime \prime}, y^{\prime \prime}, z^{\prime \prime} ; \omega\right)=e^{q^{\prime} z^{\prime \prime}} V_{t o t}\left(x^{\prime \prime}, y^{\prime \prime}, q^{\prime} ; \omega\right)$. The result is

$$
\begin{aligned}
V_{i n}\left(x, y, q^{\prime} ; \omega\right)= & \frac{2 e^{2}}{\epsilon_{b} N^{2}} \sum_{k^{\prime}} \sum_{n n^{\prime}} \sum_{t t^{\prime}} \sum_{l l^{\prime}} \prod_{\substack{n t^{\prime} \\
t t^{\prime}}}\left(k, k^{\prime} ; \omega\right) e^{-i\left(q^{\prime}-q\right) l d} e^{i\left(q^{\prime}-q\right) l^{\prime} d} \\
& \times \int d x^{\prime} \int d y^{\prime} K_{0}\left(q^{\prime}\left|\bar{r}-\bar{r}^{\prime}\right|\right) \phi_{n_{x}}^{*}\left(x^{\prime}\right) \phi_{n_{y}}^{*}\left(y^{\prime}\right) \phi_{n_{x}^{\prime}}\left(x^{\prime}\right) \phi_{n_{y}^{\prime}}\left(y^{\prime}\right) \\
& \times \int d x^{\prime \prime} \int d y^{\prime \prime} V_{t o t}\left(x^{\prime \prime}, y^{\prime \prime} ; q^{\prime}, \omega\right) \phi_{n_{x}^{\prime}}^{*}\left(x^{\prime \prime}\right) \phi_{n_{y}^{\prime}}^{*}\left(y^{\prime \prime}\right) \phi_{n_{x}}\left(x^{\prime \prime}\right) \phi_{n_{y}}\left(y^{\prime \prime}\right) \\
& \times \int d z^{\prime} e^{-i q^{\prime}\left(z^{\prime}-l d\right)} \chi_{t}^{*}\left(z^{\prime}-l d\right) \chi_{t^{\prime}}\left(z^{\prime}-l d\right) \\
& \times \int d z^{\prime \prime} e^{i q^{\prime}\left(z^{\prime \prime}-l^{\prime} d\right)} \chi_{t^{\prime}}^{*}\left(z^{\prime \prime}-l^{\prime} d\right) \chi_{t}\left(z^{\prime \prime}-l^{\prime} d\right),
\end{aligned}
$$

where $\bar{r}$ and $\bar{r}^{\prime}$ are the $2 \mathrm{D}$ vectors in the $\mathrm{x}-\mathrm{y}$ plane, $K_{0}(x)$ is the zeroth-order modified Bessel function of the second kind, which diverges as $-\ln (x)$ when $x \rightarrow 0$, and

$$
\Pi_{\substack{n n^{\prime} \\ t t^{\prime}}}\left(k, k^{\prime} ; \omega\right)=\sum_{k} \Lambda_{\substack{n n^{\prime} \\ t t^{\prime}}}\left(k, k^{\prime} ; \omega\right)=2 \sum_{k} \frac{f\left(\epsilon_{n t}\right)-f\left(\epsilon_{n^{\prime} t^{\prime}}\right)}{\epsilon_{n t}-\epsilon_{n^{\prime} t^{\prime}}+\hbar \omega^{*}}
$$

Now, it is convenient to simplify a few steps before we proceed further. (i) The last two integrals in Eq. (13) can be written as: $\left|\int d z e^{-i q^{\prime} z} \chi_{t}^{*}(z) \chi_{t^{\prime}}(z)\right|^{2}$. (ii) The sum over $l^{\prime}$ is worked out as follows:

$$
\begin{aligned}
\frac{1}{N} \sum_{l^{\prime}} e^{i\left(q-q^{\prime}\right)\left(l^{\prime}-l\right) d} & =\frac{1}{N d} \int d\left(l^{\prime} d\right) e^{i\left(q-q^{\prime}\right)\left(l^{\prime}-l\right) d} \\
& =\frac{1}{N d} 2 \pi \delta\left(q-q^{\prime}\right)=\frac{1}{L_{z}} 2 \pi \delta\left(q-q^{\prime}\right)
\end{aligned}
$$


(iii) The remaining sum over $l$ reads as follows: $\sum_{l} 1=N$. This cancels the remaining $N$ in the denominator. (iv) Then open up the sum over $k^{\prime}$ to write, with the aid of Eq. (15),

$$
\begin{aligned}
\sum_{k^{\prime}} \prod_{\substack{n n^{\prime} \\
t t^{\prime}}}\left(k, k^{\prime} ; \omega\right) \frac{2 \pi}{L_{z}} \delta\left(q-q^{\prime}\right) & =\int d k^{\prime} \Pi_{\substack{n n^{\prime} \\
t t^{\prime}}}\left(k, k^{\prime} ; \omega\right) \delta\left(k^{\prime}-k-q^{\prime}\right) \\
& =\prod_{\substack{n t \\
n^{\prime} t^{\prime}}}\left(k, k^{\prime}=k+q^{\prime} ; \omega\right)
\end{aligned}
$$

(v) Finally, in the light of Eqs. (14) - (16), one can safely replace $q^{\prime}$ by $q$ on both sides of Eq. (13) to write

$$
\begin{aligned}
V_{i n}(x, y, q ; \omega)= & \frac{2 e^{2}}{\epsilon_{b}} \sum_{n n^{\prime}} \sum_{t t^{\prime}} \Pi_{n n^{\prime}}\left(k, k^{\prime}=k+q ; \omega\right) \\
& \times \int d x^{\prime} \int d y^{\prime} K_{0}\left(q\left|\bar{r}-\bar{r}^{\prime}\right|\right) \phi_{n_{x}}^{*}\left(x^{\prime}\right) \phi_{n_{y}}^{*}\left(y^{\prime}\right) \phi_{n_{x}^{\prime}}\left(x^{\prime}\right) \phi_{n_{y}^{\prime}}\left(y^{\prime}\right) \\
& \times \int d x^{\prime \prime} \int d y^{\prime \prime} V_{t o t}\left(x^{\prime \prime}, y^{\prime \prime} ; q^{\prime}, \omega\right) \phi_{n_{x}^{\prime}}^{*}\left(x^{\prime \prime}\right) \phi_{n_{y}^{\prime}}^{*}\left(y^{\prime \prime}\right) \phi_{n_{x}}\left(x^{\prime \prime}\right) \phi_{n_{y}}\left(y^{\prime \prime}\right) \\
& \times\left|\int d z e^{-i q z} \chi_{t}^{*}(z) \chi_{t^{\prime}}(z)\right|^{2}
\end{aligned}
$$

Next, we take the matrix elements of both sides between the states $\left|m_{x}, m_{y}>\equiv\right| m>$ and $\left|m_{x}^{\prime}, m_{y}^{\prime}>\equiv\right| m^{\prime}>$ to write

$$
\begin{aligned}
<m^{\prime}\left|V_{i n}(\ldots)\right| m>=\sum_{n n^{\prime}} \sum_{t t^{\prime}} & \prod_{\substack{n n^{\prime} \\
t t^{\prime}}}\left(k, k^{\prime}=k+q ; \omega\right) U_{n n^{\prime} m m^{\prime}}(q) \\
& \times S_{t t^{\prime}}(q)<n^{\prime}\left|V_{t o t}(\ldots)\right| n>
\end{aligned}
$$

where

$$
\begin{gathered}
U_{n n^{\prime} m m^{\prime}}(q)=\frac{2 e^{2}}{\epsilon_{b}} \int d \bar{r} \int d \bar{r}^{\prime} \phi_{n}^{*}(\bar{r}) \phi_{n^{\prime}}(\bar{r}) K_{0}\left(q\left|\bar{r}-\bar{r}^{\prime}\right|\right) \phi_{m^{\prime}}^{*}\left(\bar{r}^{\prime}\right) \phi_{m}\left(\bar{r}^{\prime}\right) \\
S_{t t^{\prime}}(q)=\left|\int d z e^{-i q z} \chi_{t}^{*}(z) \chi_{t^{\prime}}(z)\right|^{2}
\end{gathered}
$$

and

$$
<n^{\prime}\left|V_{t o t}(\ldots)\right| n>=\int d \bar{r} \phi_{n^{\prime}}^{*}(\bar{r}) V_{t o t}(\bar{r} ; q, \omega) \phi_{n}(\bar{r})
$$

Notice that the quantities in Eq. (18) have been defined in Eqs. (19) - (21) in the condensed notations for the sake of brevity. Here $U_{n n^{\prime} m m^{\prime}}(q)$ is the matrix element of the Fourier-transformed Coulombic interactions. Next, let us invoke the condition of self-consistency $\left[V_{t o t}=V_{e x}+V_{i n}\right]$ on Eq. (18) to write 


$$
\begin{aligned}
& <m^{\prime}\left|V_{e x}(\ldots)\right| m>=\sum_{n n^{\prime}}\left[\delta_{n m} \delta_{n^{\prime} m^{\prime}}-\sum_{t t^{\prime}} \underset{\substack{n n^{\prime} \\
t t^{\prime}}}{\prod^{\prime}}\left(k, k^{\prime}=k+q ; \omega\right) U_{n n^{\prime} m m^{\prime}}(q)\right. \\
& \left.\times S_{t t^{\prime}}(q)\right]<n^{\prime}\left|V_{t o t}(\ldots)\right| n>
\end{aligned}
$$

Here $\delta_{n m}$ is the Kronecker delta function. Now, since the external potential and the total potential are correlated through the nonlocal, dynamic dielectric function $\epsilon\left(\bar{r}, \bar{r}^{\prime} ; \omega\right)$ such as

$$
V_{e x}(\bar{r}, \omega)=\int d \bar{r}^{\prime} \epsilon\left(\bar{r}, \bar{r}^{\prime} ; \omega\right) V_{t o t}\left(\bar{r}^{\prime}, \omega\right)
$$

we can easily deduce from Eq. (22) that the generalized nonlocal, dynamic dielectric function is given by

$$
\epsilon_{n n^{\prime} m m^{\prime}}(q ; \omega)=\delta_{n m} \delta_{n^{\prime} m^{\prime}}-\sum_{t t^{\prime}} \prod_{\substack{n n^{\prime} \\ t t^{\prime}}}\left(k, k^{\prime}=k+q ; \omega\right) S_{t t^{\prime}}(q) U_{n n^{\prime} m m^{\prime}}(q)
$$

We call attention to the fact that Eq. (24) is the main result that could be exploited to compute a host of electronic and optical properties of the system at hand. It also plays an important role in studying, for example, the inelastic electron and light (or Raman) scattering and the transport properties if we choose to work in terms of the inverse dielectric function $\epsilon_{n n^{\prime} m m^{\prime}}^{-1}(q ; \omega)[1,43]$. As regards the computation of the excitation spectrum comprised of the single-particle and collective excitations, the zeros of the dielectric function (DF) and the poles of the inverse dielectric function (IDF) must yield exactly identical results.

\section{Symmetry and degeneracy}

The problem of the 2D harmonic oscillator offers an opportunity to show the critical connection between the symmetry and the degeneracy. To this end, we need to recognize that the subband index for the energy level is $n=n_{x}+n_{y}$, where $n_{x}=0,1,2, \ldots$ and $n_{y}=0,1,2, \ldots$, independently. For the energy level $n=0$, there is only a single eigenfunction - the ground state $-\phi_{0,0}$ [with $\left.n_{x}=0=n_{y}\right]$. The next level $n=1$ has two eigenfunctions: $\phi_{0,1}$ and $\phi_{1,0}$; therefore it is degenerate. A little further thought quickly makes it clear that still higher energy states have even greater degeneracy [i.e., there are even more eigenfunctions sharing the same eigenenergies.] Here, the source of this degeneracy is the rotational symmetry of the oscillator itself - it is called symmetry degeneracy.

To grasp the role of the symmetry, it is convenient to write the Hamiltonian operator in the cylindrical coordinates when the eigenfunction in the polar coordinates reads as $\psi(r, \phi)$. What 
becomes immediately noticeable is that the (angular) coordinate $\phi$ does not appear except as a variable for differentiation. When this happens in a classical Hamiltonian, we say it is cyclic in that coordinate and the conjugate momentum (i.e., $p_{\phi}$ ) is a constant of motion. The quantal version of this statement is that the Hamiltonian operator $\hat{H}$ commutes with the operator $\left(\hat{L_{z}}\right)$ corresponding to that momentum: i.e., $\left[\hat{H}, \hat{L_{z}}\right]=0$. It is easy enough to verify this relation, but less clear why this is so. The reason that this happens in this case is that the system [i.e., the Hamiltonian] is invariant under rotation -it is isotropic.

The degree of degeneracy $g_{n}$ can be calculated relatively easily [see, e.g., standard textbooks on quantum mechanics]. For general $N$ dimensional (isotropic) harmonic oscillator characterized by quantum number $n$, the formula for the degeneracy is given by $g_{n}=\left(\begin{array}{c}N+n-1 \\ n\end{array}\right)$. This immediately reaffirms that in the one-dimensional case each energy level corresponds to a unique quantum state and hence the system as such stands as non-degenerate.

We have seen above how the symmetry leads to the degeneracy. If the situation were somehow changed to break the symmetry, then this will break the degeneracy. This can be exemplified by considering, e.g., the anisotropic strengths of the harmonic oscillator along the $\mathrm{x}$ and $\mathrm{y}$ axes.

\section{The Wannier function and the Bloch function}

The Wannier function of a band is defined in terms of the Bloch function of the same band by

$$
\chi\left(z-z_{n}\right)=\frac{1}{\sqrt{N}} \sum_{k} e^{-i k z_{n}} \phi_{k}(z),
$$

where $z_{n}$ is the spatial point in the lattice with period $d$. For the Bloch function

$$
\phi_{k}(z)=e^{i k z} u(z)
$$

the Wannier function is

$$
\chi\left(z-z_{n}\right)=\sqrt{N} u(z) \frac{\sin \left[\pi\left(z-z_{n}\right) / d\right]}{\left[\pi\left(z-z_{n}\right) / d\right]},
$$

where $u(z)=u(z+d)$ is the solution of the Schrödinger equation [see Appendix A]

$$
\left[-\frac{\hbar^{2}}{2 m^{*}} \frac{d^{2}}{d z^{2}}+V(z)\right] u(z)=\epsilon_{0} u(z)
$$

The foregoing diagnosis tells us how the kronig-Penney (KP) wave function $u(z)$, the Bloch function $\phi_{k}(z)$, and the Wannier function $\chi\left(z-z_{n}\right)$ are very tightly interwoven with each other. Since the characteristic eigenfunction in the present problem is defined in terms of these functions [see Eq. (1)], we choose to study their graphic behavior. This is illustrated in Fig. 2. We believe it 
unnecessary to expand on the obviously correct behavior of each function, since, as they say, $a$ picture is worth a thousand words.
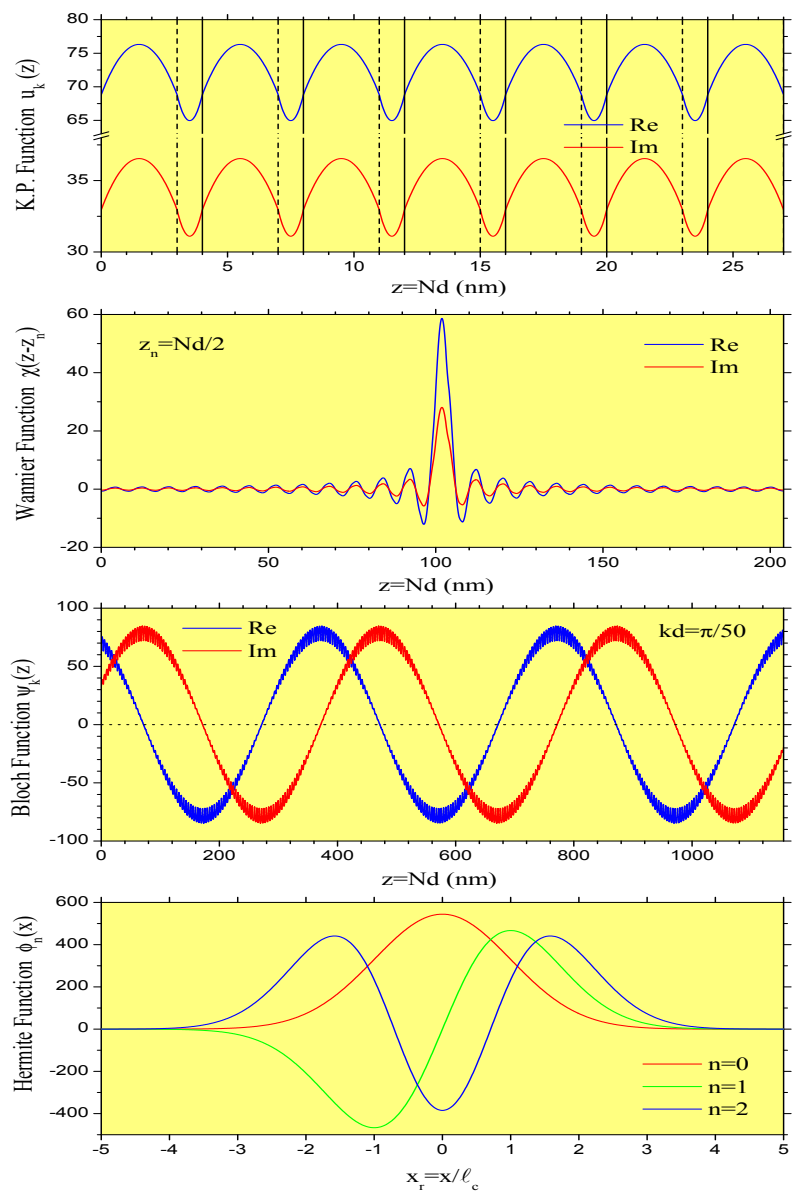

FIG. 2: (Color online) Graphic behavior of the KP, the Wannier, the Bloch, and the Hermite functions as used in this work. The well (barrier) thickness is 3 (1) nm and $V_{0}=349.11 \mathrm{meV}$.

\section{E. Miniband structure due to the BBC}

The introduction of the a superlattice potential perturbs the band structure of the host materials. The degree of such perturbation depends very much on its amplitude and the periodicity. Since the superlattice period is usually larger than the lattice constants of the (bulk) host constituents, the Brillouin zone is divided into minizones giving rise to a series of narrow allowed bands separated by forbidden regions at the zone boundaries $(k d=n \pi)$. The allowed bands and forbidden regions are known, respectively, as minibands and minigaps in the language of solid state physics. The band-gap engineering - the process of controlling the band-gap by altering the composition of the host components - is a subject of paramount importance both for fundamental 
physics and device applications.

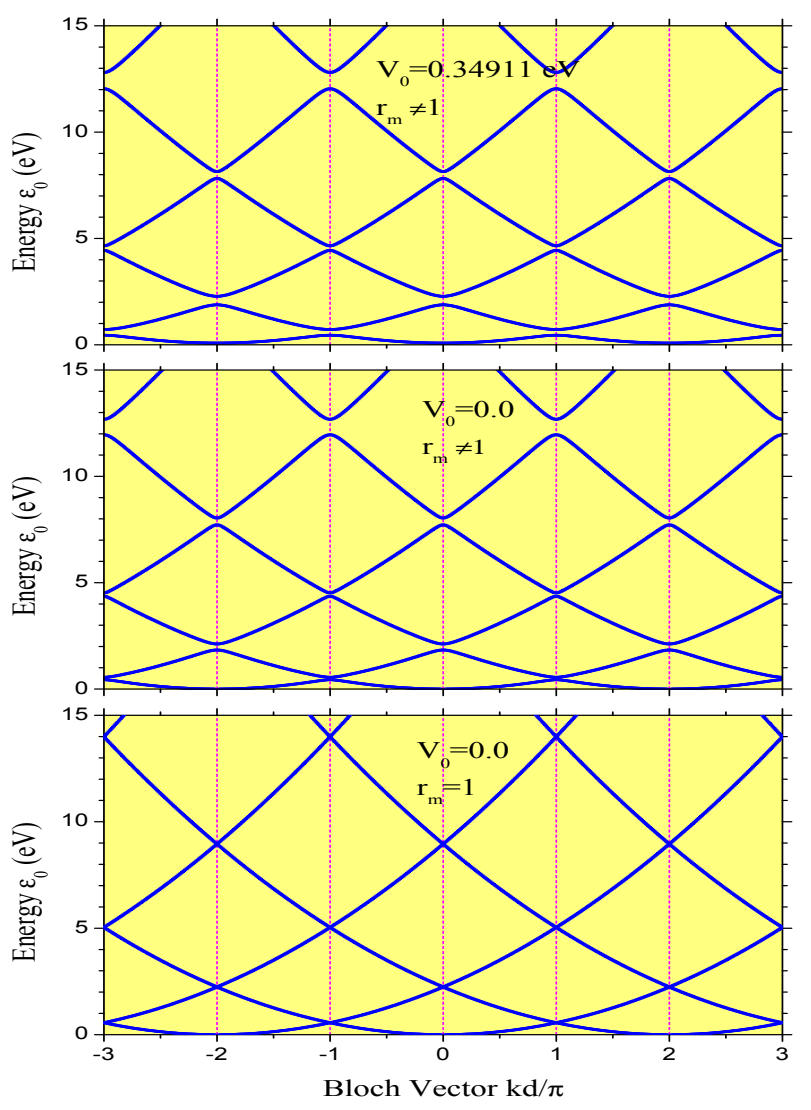

FIG. 3: (Color online) The miniband spectrum: the (KP) energy vs. the reduced Bloch vector. The top panel represents the superstructure with a finite potential barrier height $\left[V_{0}=349.11 \mathrm{meV}\right]$ and the effective mass ratio $r_{m} \neq 1$. The middle panel stands for the "free particle" $\left[V_{0}=0\right]$ with $r_{m} \neq 1$. The bottom panel refers to the "free particle" with $r_{m}=1$. We draw attention to the "free" particle observing the gaps at the zone boundaries $(k d=n \pi)$ [see the middle panel].

It is known that a free particle (electron or hole) does not observe any gap in the band structure at the zone boundaries. Yet, it turns out that in a superstructure with heterointerfaces, a "free" electron $\left(V_{0}=0\right)$ can (and does) experience gaps at the zone boundaries provided that the dispersion relation is derived by employing the proper effective-mass boundary conditions becoming known as the BBC. This is illustrated in Fig. 3 (see, e.g., the middle panel). This implies that a "free" electron virtually feels an effective potential barrier due, in fact, to the dissimilar hosts (with different $m^{*}$ ) in the wells and the barriers. It is noteworthy, however, that the magnitude of the gaps for the free particle (the middle panel) is relatively smaller than that for the bound particle (top panel). 


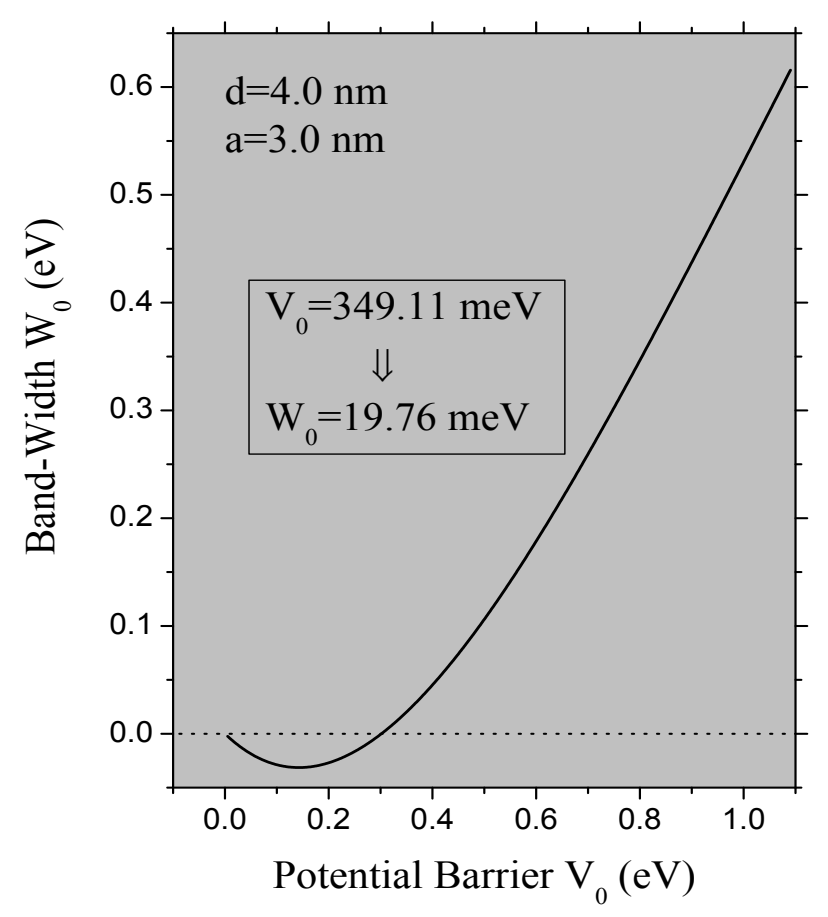

FIG. 4: The bandwidth of the (lowest) miniband as a function of potential barrier height $V_{0}$. The rest of the parameters are as listed inside the picture.

\section{F. On the significance of the bandwidth}

A superlattice (periodic) potential gives rise to the miniband spectrum because of the quasifree motion of the charge carriers along the growth axis. The bandwidth of these minibands can be controlled by varying, e.g., the composition of the superstructure, temperature, electric field, and magnetic field. At the same time, the bandwidth can have significant control over numerous electronic, optical, and transport phenomena. This is because the bandwidth is, in part, a measure of the electron dynamics along the superlattice axis. If the ratio of the barrier height to the miniband bandwidth is much smaller than unity, electrons can roam almost freely and influence the degree of tunneling. On the other hand, if this ratio is much greater than unity, electrons are mostly confined within the quantum-dot islands. Since the bandwidth in the present problem governs the excitation spectrum [see, e.g., Eq. (4)], we thought it worthwhile to study the bandwidth as function of the potential barrier. This is shown in Fig. 4. It deserves mention that we choose to work with a bandwidth $W_{0}(=19.76 \mathrm{meV})<\hbar \omega_{\text {opt }}(\gtrsim 30 \mathrm{meV})$ so as to avoid the optical phonon scattering. While it is not of vital interest here, we notice that the bandwidth $W_{0}$ attains a negative value for the barrier height in the range $0.00521 \lesssim V_{0}(\mathrm{eV}) \lesssim 0.30099$. Energy bands with positive (negative) frequencies with respect to the (chosen) zero of the energy scale 
refer to the positive (negative) bandwidths. For real signals, the negative frequencies are always present and are always a mirror of the positive frequencies.

\section{G. On limiting the number of subbands}

Equation (24) is, in general, an $\infty \times \infty$ matrix until and unless we cut off the number of subbands (and hence limit the electronic transitions) involved in the problem. First of all, we limit ourselves to the lowest miniband (i.e., $t, t^{\prime} \equiv 0$ ). Next, it is noteworthy that while experiments may report multiple subbands occupied, theoretically it is not only extremely difficult but also (almost) impossible to compute the excitation spectrum for the multiple-subband model. This is because the generalized dielectric function turns out to be a matrix of the dimension of $\eta^{2} \times \eta^{2}$, where $\eta$ is the number of subbands in the model. Handling such enormous matrices (for a very large $\eta$ ) analytically is a hard nut to crack and if someone wants to do it numerically then what one is ultimately left with is just a numerical simulation, which, generally, becomes a matter out of taste for someone who likes to seek an understandable correspondence between the analytical and the numerical results. For this reason, we choose to keep the complexity to a minimum and limit ourselves to a two-subband model $\left(n, n^{\prime}, m, m^{\prime} \equiv 1,2\right)$ with only the lowest one occupied. This is known to be quite a reasonable assumption for these low-density, lower-dimensional (quantum) systems at lower temperatures where most of the (electronic, optical, and transport) experiments are performed. This implies that the generalized dielectric function is to be a $4 \times 4$ matrix. First, let us cast Eq. (24) in the following form.

$$
\epsilon_{n n^{\prime} m m^{\prime}}(q ; \omega)=\delta_{n m} \delta_{n^{\prime} m^{\prime}}-A_{n n^{\prime}}(q, \omega) P_{n n^{\prime} m m^{\prime}}(q),
$$

where

$$
A_{n n^{\prime}}(q, \omega)=\Pi_{n n^{\prime}}\left(k, k^{\prime}=k+q ; \omega\right) S_{00}(q),
$$

where $S_{00}(q)$ is just as defined in Eq. (20) [with $t, t^{\prime} \equiv 0$ ] and

$$
P_{n n^{\prime} m m^{\prime}}(q)=\int d \bar{r} \int d \bar{r}^{\prime} \phi_{n}^{*}(\bar{r}) \phi_{n^{\prime}}^{*}(\bar{r}) V_{e e}\left(q, \bar{r}-\bar{r}^{\prime}\right) \phi_{m^{\prime}}^{*}\left(\bar{r}^{\prime}\right) \phi_{m}\left(\bar{r}^{\prime}\right),
$$

where

$$
V_{e e}\left(q, \bar{r}-\bar{r}^{\prime}\right)=\frac{2 e^{2}}{\epsilon_{b}} K_{0}\left(q\left|\bar{r}-\bar{r}^{\prime}\right|\right)
$$


Now, the generalized dielectric function matrix in Eq. (29) takes the following form.

$$
\tilde{\epsilon}(q, \omega)=\left[\begin{array}{cccc}
1-A_{11} P_{1111} & -A_{11} P_{1112} & -A_{11} P_{1121} & -A_{11} P_{1122} \\
-A_{12} P_{1211} & 1-A_{12} P_{1212} & -A_{12} P_{1221} & -A_{12} P_{1222} \\
-A_{21} P_{2111} & -A_{21} P_{2112} & 1-A_{21} P_{2121} & -A_{21} P_{2122} \\
-A_{22} P_{2211} & -A_{22} P_{2212} & -A_{22} P_{2221} & 1-A_{22} P_{2222}
\end{array}\right]
$$

Notice that $A_{22}=0$ because the second subband is unoccupied. Then, since the quasi-particle excitations are given by $|\tilde{\epsilon}(q, \omega)|=0$, Eq. (33) finally yields

$$
\left(1-A_{11} P_{1111}\right)\left(1-B_{12} P_{1212}\right)-A_{11} B_{12} P_{1112}^{2}=0
$$

where $B_{12}=A_{12}+A_{21}=\chi_{12} S_{00}$ (with $\chi_{12}=\Pi_{12}+\Pi_{21}$ ) is the intersubband polarizability function that accounts for upward as well as downward transitions. This is the final equation which has to be treated at the computational level. However, further simplification is possible depending on the nature of the confining potential (see next).

\section{H. On the symmetry of the confining potential}

It is becoming known [1] that for a symmetric potential well, $P_{i j k l}$ (the Fourier-transformed Coulomb interaction) is strictly zero provided that $i+j+k+l$ is an odd number. This is so because the corresponding wave function is either symmetric or antisymmetric under space reflection. Since the two-dimensional parabolic confining potential in the InAs islands is symmetric, $P_{1112}=0$ in Eq. (34). This tells us that the intrasubband and intersubband modes represented, respectively, by the first and second factors in the first term in Eq. (34) are decoupled, because the (second) coupling term is zero. That $P_{1112}=0$ can be immediately verified just by rewriting Eq. (31) in the polar coordinates. Since the subband index 0 (1) is allowed for the intrasubband (intersubband) excitation, we still need to conform Eq. (34) such that the subscript $1 \rightarrow 0$ and $2 \rightarrow 1$ for all practical purposes.

\section{The zero-temperature limit}

Since extremely low-temperatures are preferred for the experiments performed on the lower dimensional systems, we choose to confine ourselves to the zero temperature limit. We think the temperature dependence of our results would be significant only at $T \gtrsim 35 \mathrm{~K}$. In that situation, 
we can change the sum (over $k$ ) to an integral by using the replacement rule and replace the Fermi distribution function with the Heaviside unit step function such that

$$
f(\epsilon)=\theta\left(\epsilon_{F}-\epsilon\right)=\left\{\begin{array}{lll}
1 & \text { if } & \epsilon_{\mathrm{F}}>\epsilon \\
0 & \text { if } & \epsilon_{\mathrm{F}}<\epsilon
\end{array},\right.
$$

where $\epsilon_{F}$ is the Fermi energy in the problem. It is interesting to notice that in the zero-temperature limit, we are allowed to go further to calculate analytically the polarizability functions $\Pi_{00}$ and $\chi_{01}$. To this end, we first need to simplify the sum over $k$ [in, e.g., Eq. (14)]. This results in a definite integral with the upper (lower) limit as $k_{F}\left(-k_{F}\right)$. Given the multiple definitions of that integral (and the lack of a single definitive source) [45], we think it is more important to define here that integral instead of writing the (lengthy) expressions of $\Pi_{00}$ and $\chi_{01}$. The integral reads as

$$
\begin{aligned}
\int d x & \frac{1}{[a+b \cos (x)+c \sin (x)]} \\
& =\frac{2}{\sqrt{+a^{2}-b^{2}-c^{2}}} \tan ^{-1}\left[\frac{c+(a-b) \tan (x / 2)}{\sqrt{+a^{2}-b^{2}-c^{2}}}\right], \quad \mathrm{a}^{2}>\mathrm{b}^{2}+\mathrm{c}^{2} \\
& =\frac{-2}{\sqrt{-a^{2}+b^{2}+c^{2}}} \tanh ^{-1}\left[\frac{c+(a-b) \tan (x / 2)}{\sqrt{-a^{2}+b^{2}+c^{2}}}\right], \quad \mathrm{a}^{2}<\mathrm{b}^{2}+\mathrm{c}^{2} ;|\mathrm{y}|<1 \\
& =\frac{-2}{\sqrt{-a^{2}+b^{2}+c^{2}}} \operatorname{coth}^{-1}\left[\frac{c+(a-b) \tan (x / 2)}{\sqrt{-a^{2}+b^{2}+c^{2}}}\right], \quad \mathrm{a}^{2}<\mathrm{b}^{2}+\mathrm{c}^{2} ;|\mathrm{y}|>1
\end{aligned}
$$

where $y=$ [the whole quantity within the square brackets in the respective equality]. The symbols $a, b, c$ are not to be confused with the well/barrier widths. Although one can analytically convert one form into the other, the computer does not know this. What follows next is to express the hyperbolic functions in terms of the logarithmic ones in order to obtain the manageable forms of $\Pi_{00}$ and $\chi_{01}$. We analyze the resulting forms of $\Pi_{00}$ and $\chi_{01}$ in the long wavelength limit to obtain

$$
\Pi_{00}=\frac{n_{1 D} q^{2}}{m_{e}^{*} \omega^{2}}+O\left(q^{4}\right)
$$

where $m_{e}^{*}\left[=\hbar^{2} /\left(W_{0} d^{2}\right)\right]$ stands for an effective mass in the problem, and

$$
\chi_{01}=\frac{2 n_{1 D} \epsilon_{21}}{(\hbar \omega)^{2}-\epsilon_{21}^{2}}+O(q)
$$

where $\epsilon_{21}=\hbar \omega_{0}$ is the subband spacing. It is interesting to note that the long wavelength forms of the polarizability functions derived in Eqs. (37) and (38) are independent of the dimensionality of the system and have the same forms for higher dimensions as well [1]. 


\section{J. The Density of states and the Fermi energy}

An analytical treatment requires that we start with Eq. (4). We derive the following expression for computing self-consistently

$$
g(\epsilon)=\frac{2}{\pi d} \sum_{n}\left[W_{h}^{2}-\left(\epsilon-\epsilon_{n}\right)^{2}\right]^{-1 / 2} \theta\left[W_{h}^{2}-\left(\epsilon-\epsilon_{n}\right)^{2}\right],
$$

the density of states (DOS), and

$$
n_{1 D}=\frac{2}{\pi d} \sum_{n} \cos ^{-1}\left[-\frac{1}{W_{h}}\left(\epsilon_{F}-\epsilon_{n}\right)\right] \theta\left[-\frac{1}{W_{h}}\left(\epsilon_{F}-\epsilon_{n}\right)\right],
$$

the Fermi energy. Here $W_{h}=W_{0} / 2, \epsilon_{n}=(n+1) \hbar \omega_{0}+\epsilon_{0}$, and $n_{1 D}$ is the linear charge density of the electrons (i.e., the number of electrons per unit length). Note that $\epsilon_{0}$ is the energy of the lowest miniband at the zone center and can be neglected with no loss of generality (because it affects neither the intra- nor the inter-subband excitations).

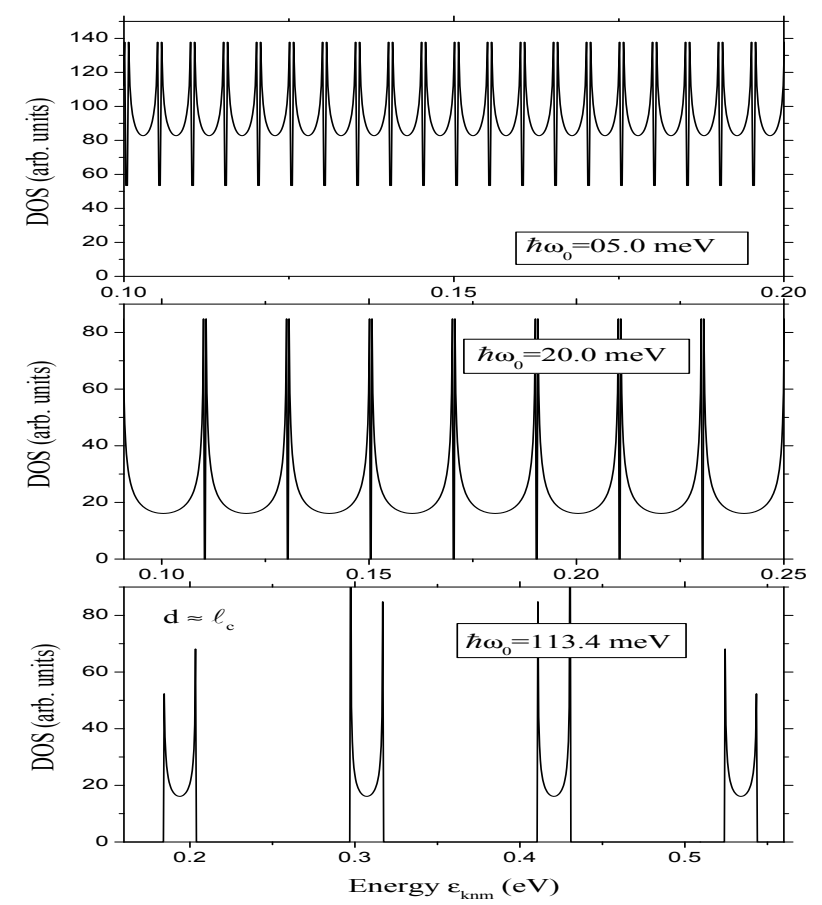

FIG. 5: The density of states vs. the excitation energy for the resultant quantum wire. The confinement potential (or subband spacing) is defined as $\hbar \omega_{0}=5.0 \mathrm{meV}$ (top panel), $20.0 \mathrm{meV}$ (middle panel), and $113.4 \mathrm{meV}$ (bottom panel). The subband spacing in the bottom panel was designed to have $\ell_{c} \simeq d$. The band-width is $W_{0}=19.76 \mathrm{meV}$.

Figure 5 depicts the results on the density of states versus the excitation energy. We observe that as the confinement increases the DOS structure (in the top panel) is pushed downwards to 
take finally the shape shown in the middle panel where all the spikes visible in the upper panel start from the origin. With still stronger confinement, the structure in the DOS starts splitting in energy as seen in the bottom panel, where the resultant system favors the decoupled InAs islands. In other words, very strong confinement seems to prevent the forming of the quantum wire due, virtually, to little quasi-free motion along the growth direction. This also reaffirms the fact that the DOS is very much a dimensionality dependent property of the system and the role dimensions play is evident from the units of the DOS: $g(\epsilon) \propto \epsilon^{1 / 2}$ (in 3D), $g(\epsilon) \propto \epsilon^{0}$ (in 2D), $g(\epsilon) \propto \epsilon^{-1 / 2}$ (in 1D). In three-dimensionally confined quantum dots the DOS is known to be $\delta$-function-like leading to the well-argued vanishing of the thermal broadening. We had purposely designed the confinement in the bottom panel in order to have a competition between the period $(d)$ and the characteristic length of the harmonic oscillator $\left(\ell_{c}\right)$ [i.e., to have $d \simeq \ell_{c}$ ]. However, we did not observe any specific effect due to this condition.

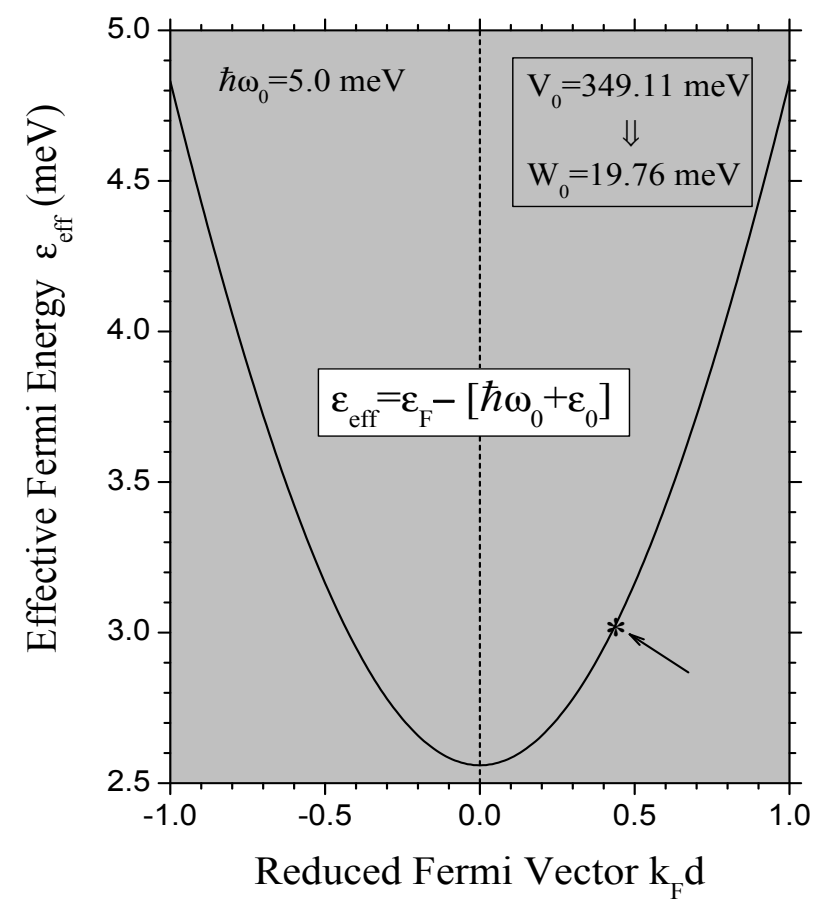

FIG. 6: The Fermi energy vs. the reduced Fermi vector for the resultant quantum wire. We purposely made this choice to have $k_{F} d$ on the abscissa - it could have been simply the charge density $n_{1 D}$. The subband spacing $\hbar \omega_{0}=5.0 \mathrm{meV}$ and the band-width $W_{0}=19.76 \mathrm{meV}$. The $\star$ on the Fermi energy curve corresponds to $\epsilon_{e f f}=3.0296 \mathrm{meV}$.

Figure 6 illustrates the (effective) Fermi energy as a function of the dimensionless Fermi vector $\left(k_{F} d\right)$. Since the Fermi vector is related to the $1 \mathrm{D}$ charge density by means of $k_{F}=(\pi / 2) n_{1 D}$, one could choose to plot the Fermi energy versus the charge density. However, we made this choice so 
as to feel free in choosing, e.g., $n_{1 D}$ and $d$ simultaneously. The Fermi energy curve turns out to be symmetric because the Fermi vector (or the Fermi number) happens to be inside the argument of a cosine term in the single-particle energy. The $\star$ on the Fermi energy curve refers to the effective Fermi energy $\epsilon_{e f f}=3.0296 \mathrm{meV}$ - a value that corresponds to $k_{F} d=0.4398$. This implies that $n_{1 D}=0.7 \times 10^{6} \mathrm{~cm}^{-1}$, for a period of $d=4.0 \mathrm{~nm}$. We will see in the next section that our choice of the subband spacing $\hbar \omega_{0}=5.0 \mathrm{meV}$ in a two-subband model (with only the lowest one occupied) is justifiable since the Fermi energy, for this set of parameters, lies at $\epsilon_{e f f}=3.0296 \mathrm{meV}$ (i.e., $\left.0<\epsilon_{e f f}<\hbar \omega_{0}\right)$. The chemical potential at zero temperature is equal to the Fermi energy. The knowledge of the variation of the Fermi energy is of cardinal importance to the understanding of (almost all) electronic, optical, and transport phenomena in a quantal system. This is true to the extent that the transport properties (such as conductance, resistance, ..etc.) are reflections of the electron dynamics at/near the Fermi surface in the system. The fascinating thing about the Fermi surface is that you can tailor it before it tailors the rest.

\section{K. On the behavior of $S_{00}(q)$}

In this subsection, we would like to see how the factor $S_{00}(q)$, which virtually governs the quasi-free motion along the growth direction, behaves as the momentum transfer $q$ and/or the well-width $a$ varies. This is shown in Fig. 7, where we plot $S_{00}(q)$ as a function of momentum transfer $q / k_{F}$ (in left panel) and as a function of well-width $a$ (in the right panel). As seen in the left panel, $S_{00}$ starts with a finite value at the zone center and decreases monotonously so as to form a half inverted parabola centered at its starting value. When we fix the momentum transfer and vary the well-width, the factor $S_{00}$ again starts from a nonzero value at $a \simeq 2.78$ $\mathrm{nm}$ and tends to decrease in such a manner as to make a half parabola centered at $a \simeq 8.2 \mathrm{~nm}$. Repeating this procedure for higher values of the barrier widths $b$ reveals that while the factor $S_{00}$ increases with increasing $b$, it exhibits a similar behavior as for the lower values of $b$. In addition, the factor $S_{00}$ seems to saturate at $a \simeq 8.47 \mathrm{~nm}$ for higher values of $b$. Since $S_{00}$ only contributes to the collective excitations, single-particle excitations remain unaffected by its very existence in the formulation. 

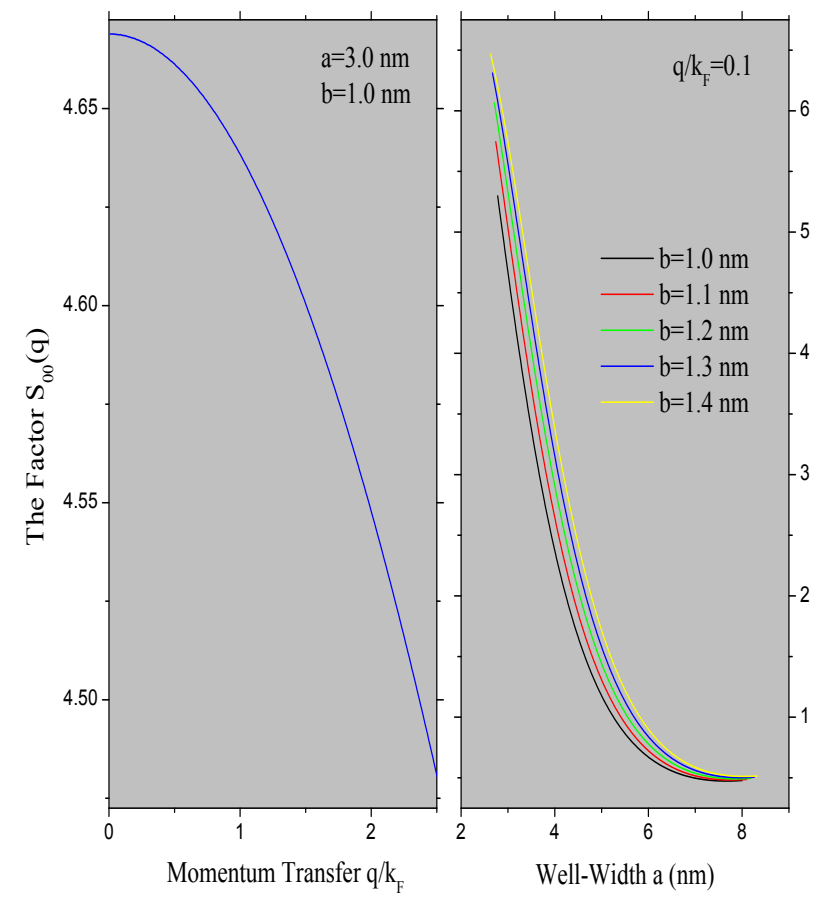

FIG. 7: (Color online) The factor $S_{00}$ vs. reduced momentum transfer $q / k_{F}$ (left panel) and vs. well-width $a$ (right panel). The parameters involved in the computation are listed inside the picture.

\section{ILLUSTRATIVE EXAMPLES ON THE PLASMON EXCITATIONS}

For the illustrative numerical examples, we focus on the InAs/GaAs system like the one in the original experiment [3]. The material parameters used are: effective mass $m^{*}=0.042 m_{0}\left(0.067 m_{0}\right)$ and the background dielectric constant $\epsilon_{b}=13.9$ (12.8) for the InAs (GaAs). We use the potential barrier of height $V_{0}=349.11 \mathrm{meV}$ that produces the band-width (of the lowest miniband) to be $W_{0}=19.76 \mathrm{meV}$, in compliance with Sakaki [2] so as to be beyond the regime of the optical phonon scattering. Further, we take the subband spacing $\hbar \omega_{0}=5.0 \mathrm{meV}$, the self-consistently determined effective Fermi energy $\epsilon_{e f f}=3.0296 \mathrm{meV}$ for a $1 \mathrm{D}$ charge density $n_{1 D}=0.7 \times 10^{6} \mathrm{~cm}^{-1}$, and the effective confinement width of the parabolic potential well, estimated as the FWHM of the extent of the Hermite function, $w_{\text {eff }}=2 \sqrt{2 \ln (2)} \sqrt{n+1} \ell_{c}=44.857 \mathrm{~nm}$. The layer widths will be specified case-wise while discussing the results. Notice that the Fermi energy $\epsilon_{F}$ varies when the charge density $\left(n_{1 D}\right)$ or the confining potential $\left(\hbar \omega_{0}\right)$ is varied. Thus we aim at exploring the single-particle and collective excitations in a quantum wire made up of vertically stacked quantum dots in the absence of an applied magnetic field at $\mathrm{T}=0 \mathrm{~K}$ in a two-subband model within the full RPA. The case of a nonzero (finite) magnetic field is deferred to a future publication. 


\section{A. The excitation spectra}

Figure 8 illustrates the full excitation spectrum composed of single-particle and collective (plasmon) excitations for a quantum wire plotted as the energy $\hbar \omega$ vs. the reduced momentum transfer $q / k_{F}$, for the given values of the charge density $n_{1 D}$, subband spacing $\hbar \omega_{0}$, and the layer thicknesses $a$ and $b$. Note that this is the case when the well width $a$ is greater than the barrier width $b$. The light (dark) shaded region refers, respectively, to the intrasubband (intersubband) single-particle excitations (SPE). The bold solid curve marked as $\Omega_{00}\left(\Omega_{10}\right)$ is the intrasubband (intersubband) collective (plasmon) excitation (CPE). The dashed curves inside and in the close vicinity of the upper edge of the SPE are also the CPE, but these modes are Landau damped and we will not be discussing them further. The intrasubband CPE starts from the origin and is not seen to merge with the upper edge of the intrasubband SPE until in the short wavelength limit. In that sense it is a bonafide, long-lived plasmon which should be easily observed in, for example, the resonant Raman scattering. The intersubband CPE starts at $\left(q / k_{F}=0, \hbar \omega=12.926 \mathrm{meV}\right)$, attains a minimum at $\left(q / k_{F}=0.61, \hbar \omega=7.73 \mathrm{meV}\right)$, and then rises up to propagate very closely to the upper edge of the intersubband SPE. However, even the instersubband CPE is not observed to merge entirely with the upper edge of the intersubband SPE. Therefore, it also remains free from Landau damping until very large propagation vector. It is not difficult to prove (analytically) why the energy of the lower branch of the intrasubband SPE goes to zero at $q=2 k_{F}$, why the lower branch of the intersubband SPE exhibits its minimum at $q=k_{F}$, and why the intersubband SPE starts at the subband spacing $(\hbar \omega=5.0 \mathrm{meV})$ at $q=0$. The most interesting aspects of this excitation spectrum are: (i) both genuine collective excitations which are free from Landau damping, (ii) the existence of the intersubband CPE which changes the sign of its group velocity before tending to merge with the respective SPE, and (iii) the overlap of the intrasubband and intersubband SPE as compared to the Figs. 9 and 10 below. The ratio of the intersubband resonance $\left(\omega_{*}\right)$ to the subband spacing $\left(\omega_{0}\right)$ at $q=0$ is found to be $\omega_{*} / \omega_{0}=2.5852$. Later we remark on this energy shift of the intersubband $\mathrm{CPE}$ with respect to the corresponding SPE.

Figure 9 shows the full excitation spectrum made up of single-particle and collective (plasmon) excitations for a quantum wire plotted as the energy $\hbar \omega$ vs. the reduced momentum transfer $q / k_{F}$ just as in Fig. 8, but for the case where the well and the barrier widths are equal (i.e., $a=b$ ). It should be pointed out that we purposely choose minimum possible widths for both the well and the barrier so that the full excitation spectrum could emerge. The importance of the variation in the layer thicknesses is remarkable. The whole spectrum has considerably decreased in energy 


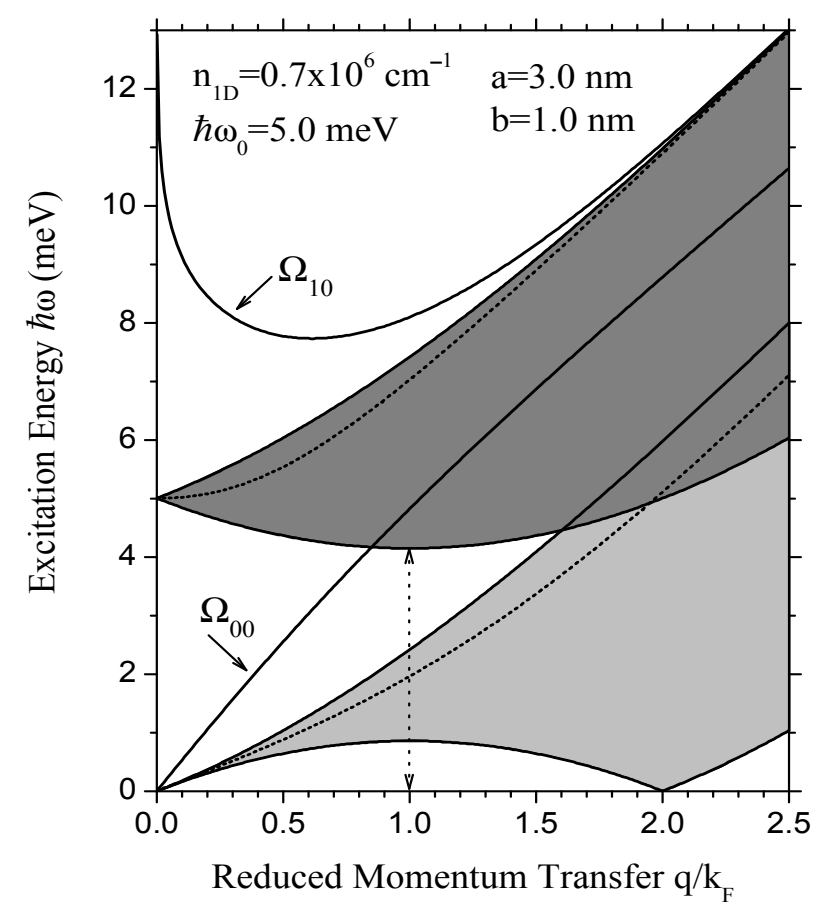

FIG. 8: The excitation spectrum of a quantum wire within a two-subband model where the energy $\hbar \omega$ is plotted as a function of the reduced momentum transfer $q / k_{F}$. The light (dark) shaded region refers to the intrasubband (intersubband) SPE associated with the lowest occupied (first excited) subband at $T=0$. The bold lower (upper) curve represents the intrasubband (intersubband) CPE. The dashed curves inside the SPE are the respective Landau damped CPE with no life-time at all. The vertical double-headed arrow stresses the minimum of the intersubband SPE at $q=k_{F}$. We call attention to the intersubband $\mathrm{CPE}$ which changes the sign of its group velocity before tending to merge with the respective SPE. The relevant parameters are as listed inside the picture. It is noteworthy that the $a>b$.

as compared to that in Fig. 8. There is a wide energy gap between the intersubband and the intrasubband excitations. Apart from the fact that it now starts at lower energy $(\hbar \omega=6.7094$ $\mathrm{meV}$ ), the intersubband collective (plasmon) excitation has now smoothed out its wide dip present in Fig. 8. In addition, the intersubband CPE is now seen to merge with the upper edge of the corresponding SPE at $q / k_{F} \simeq 2.17$ and hence becomes Landau-damped thereafter. The ratio $\omega_{*} / \omega_{0}$ is now defined as $\omega_{*} / \omega_{0}=1.3418$. The rest of the discussion related with Fig. 8 is still valid.

Figure 10 depicts the full excitation spectrum comprised of single-particle and collective (plasmon) excitations for a quantum wire plotted as the energy $\hbar \omega$ vs. the reduced momentum transfer $q / k_{F}$ just as in Fig. 8, but for the case where the well and the barrier widths are such that $a<b$. Again while intending to keep the barrier width greater than the well width, we kept to choose 


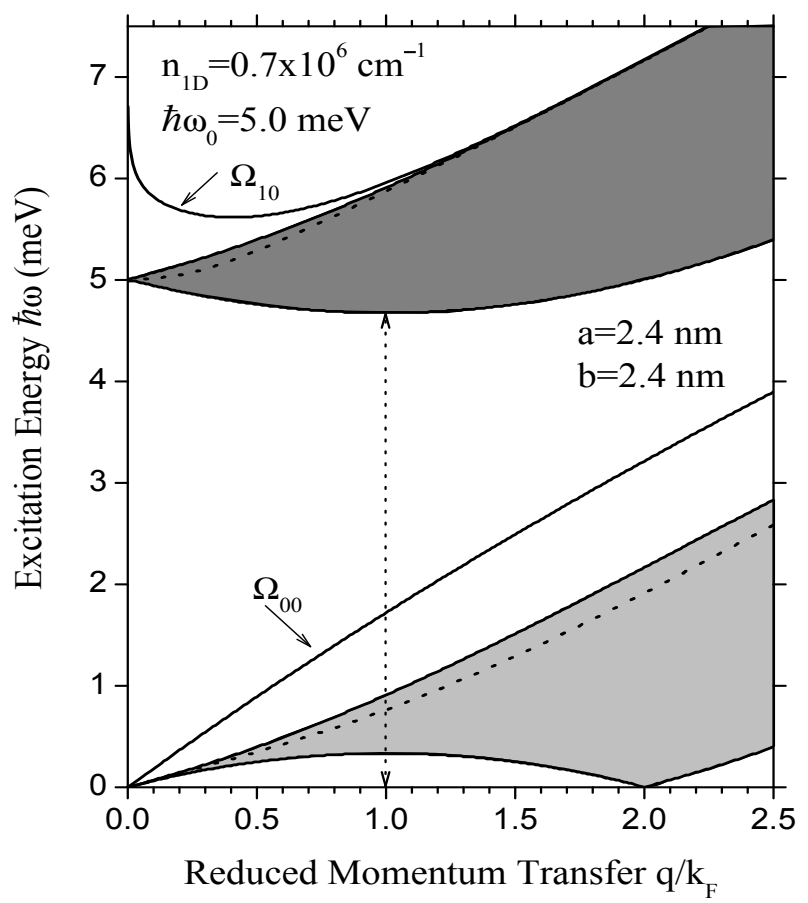

FIG. 9: The same as in Fig. 8, but for the case where the well and the barrier widths are equal (i.e., $a=b)$. We call attention to lowering of the whole spectrum in energy and smoothing out of the big dip in the intersubband CPE in Fig. 8. The relevant parameters are as listed inside the picture.

the minimum $a$ and $b$. We observe further lowering of the whole spectrum in energy and a wider gap between the intersubband and intrasubband excitations as compared to Fig. 9. It is, however, interesting to note that the intersubband CPE does not merge with the upper branch of the respective SPE and remains free from Landau damping, unlike Fig. 9. The ratio $\omega_{*} / \omega_{0}$ is now given by $\omega_{*} / \omega_{0}=1.0427$. The drastic differences observed in the excitation spectrum in Figs. 9 and 10 as compared to Fig. 8 can be intuitively understood as follows. The variation in the layer thicknesses in Figs. 9 and 10 have virtually resulted in reduction of the well width as compared to the barrier width. This implies that the wavelength of the respective excitations arising due to the charge carriers available in the wells has been implicitly enhanced and consequently the frequency (or the energy) lowered.

We think that the discussions of the excitation spectrum in Figs. 8 - 10 will remain incomplete until we shed some light on the energy shift of the intersubband CPE with respect to the corresponding SPE at $q=0$. At the momentum transfer $q=0$, the intersubband CPE generally starts at higher energy than the intersubband SPE (or the subband spacing). This shift of the intersubband resonance $\left(\hbar \omega_{*}\right)$ to an energy significantly higher than the subband spacing $\left(\hbar \omega_{0}\right)$ is a manifestation of the many-body effects such as depolarization and excitonic shifts [1]. We 


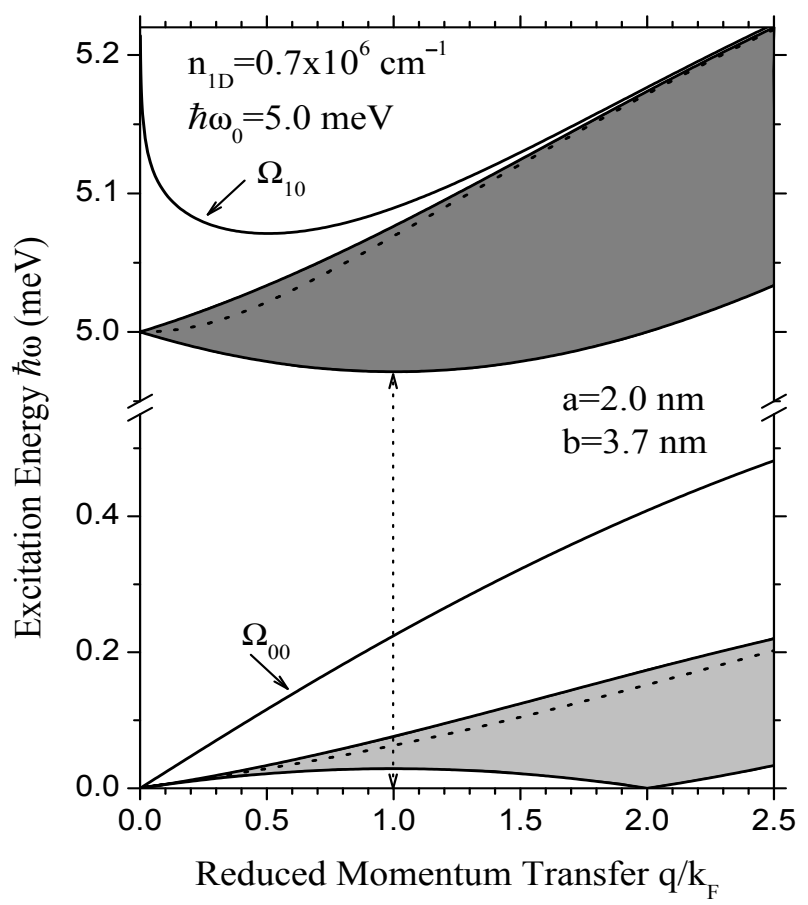

FIG. 10: The same as in Fig. 8, but for the case where the well and the barrier widths are again unequal in a manner such that now $a<b$. We call attention to still further lowering of the whole spectrum in energy and wider gap between the intersubband and intrasubband excitations as compared to Fig. 9. Notice the scale break on the energy axis. The relevant parameters are as listed inside the picture.

assume that the depolarization effects are dominant and thus have $\omega_{*}=\left(\omega_{0}^{2}+\omega_{d}^{2}\right)^{1 / 2}$, where $\omega_{d}$ is the depolarization frequency. In the absence of a desired quantal model, an upper bound on the depolarization shift can be obtained classically to yield $\omega_{d}=\sqrt{8 \pi e^{2} n_{1 D} /\left(\tilde{\epsilon} m^{*} w_{e f f}^{2}\right)}$. With our input parameters, this requires an effective (background dielectric) constant $\tilde{\epsilon}=1.11 \epsilon_{b}, 7.82 \epsilon_{b}$, and $71.64 \epsilon_{b}$, respectively, in Figs. 8, 9, and 10. This signals the importance of the screening effects in such quantum wires. A large depolarization shift such as, e.g., the one in Fig. 8 is, generally, not compensated by the excitonic shift (only reduced roughly by $20 \%$ ). The larger the ratio $\tilde{\epsilon} / \epsilon_{b}$ the stronger the screening effects and the better the chances of such compensation. In that sense the depolarization shift in Fig. 10 can be accounted for better than in Fig. 9 (and much better than in Fig. 8).

\section{B. Influence of the layer thicknesses}

Since the well and the barrier widths were seen to affect the excitation spectrum strikingly, we thought it worthwhile to study exclusively their influence on the collective excitations. Figure 11 
illustrates the intrasubband CPE plotted as a function of the barrier width $b$ for a given momentum transfer $q / k_{F}=0.05$ and for several values of the well width $a$. First of all, it is noteworthy that for $b=0$ where the system reduces to a single (homogeneous) quantum wire, the plasmon energy remains the same for all the cases as is intuitively expected. We observe that except for a small rise in energy for $b \lesssim 0.5 \mathrm{~nm}$ where the coupling is strong, the plasmon energy decreases with increasing barrier width. For smaller $b$, the larger the $a$, the higher the plasmon energy, but, for larger $b$, this trend is reversed. This behavior can also be interpreted in terms of the tunneling strength since the smaller (larger) $b$ indicates the stronger (weaker) tunneling.

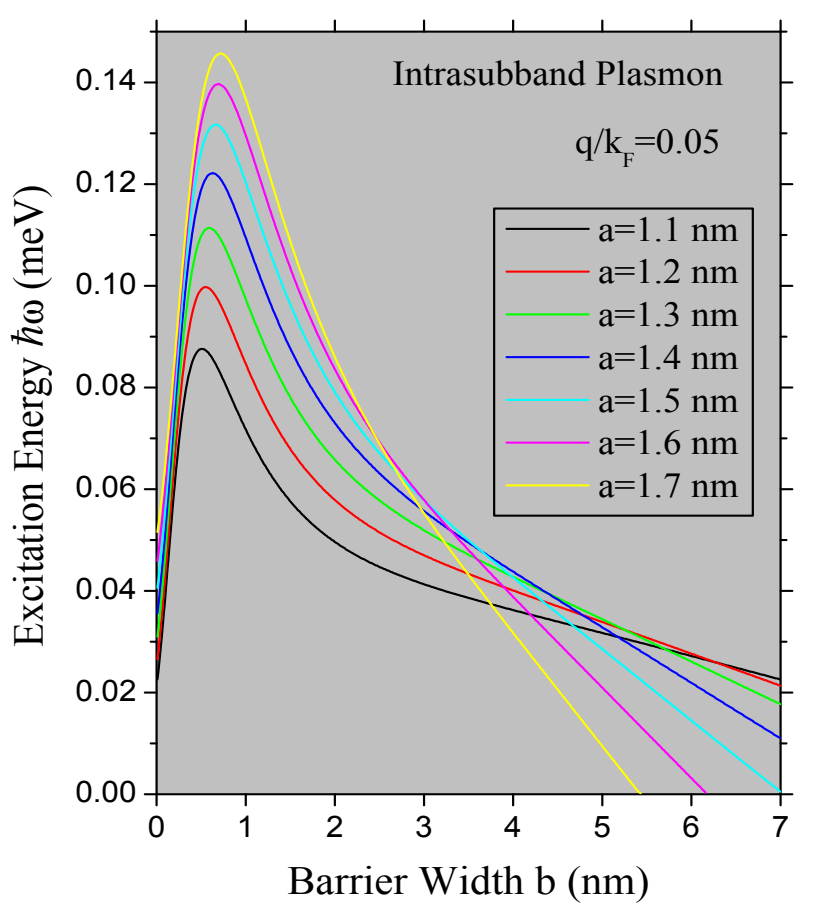

FIG. 11: (Color online) Intrasubband plasmon dispersion vs. the barrier width for several values of the well width. The momentum transfer is fixed as $q / k_{F}=0.05$. The other parameters are the same as in Fig. 8.

Figure 12 shows the intrasubband plasmon energy as a function of the well width for a given value of momentum transfer $q / k_{F}=0.05$ and for several values of barrier width. We separate the figure into two panels for the reasons of clarity: the left (right) panel covers the range $0.0<$ $a(\mathrm{~nm})<3.0(2.5<a(\mathrm{~nm})<9.0)$. It is interesting to notice that the plasmon originates with infinitesimally small (but nonzero) energy at very small value of $a$. This is what we intuitively expect. For $0.0<a(\mathrm{~nm}) \lesssim 2.75$, we observe that the smaller the $b$, the higher the plasmon energy. However, as we approach the range $2.75 \lesssim a(\mathrm{~nm})<9.0$ the preceding trend is reversed. Notice that now it is much less because of the variation in the coupling (or the tunneling) strength but 
more because of a different aspect inherent to the tight-binding approximation (TBA). As the well width becomes larger and larger the Wannier functions gradually lose their strength. As a consequence, the coupling within the neighboring wells weakens and this leads to the reversal of the trend in the latter range of the well width.

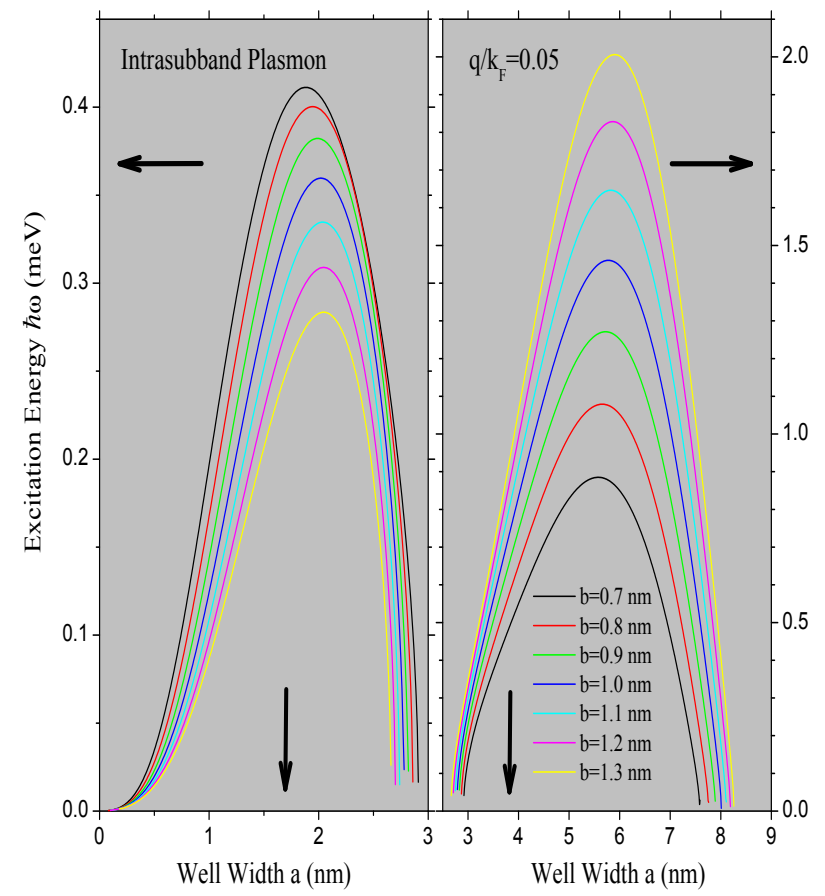

FIG. 12: (Color online) Intrasubband plasmon dispersion vs. the well width for several values of the barrier width. The momentum transfer is fixed as $q / k_{F}=0.05$. We call attention to the difference in the energy and the well-width scales between the two panels which were divided for the sake of clarity. The other parameters are the same as in Fig. 8.

Figure 13 depicts the intersubband plasmon energy as a function of the barrier width for a given value of the momentum transfer $q / k_{F}=0.1$ and for several values of the well width. Before we discuss this and the next figure, we need to consider an another issue: the existence of intersubband excitations requires the wells to be of moderate width; extremely thin wells cannot excite the intersubband excitations. Given this, we see that the plasmon energy obeys this rule in the range $0.0<b(\mathrm{~nm}) \lesssim 1.34$ : the larger the $a$, the lower the plasmon energy. This is again due to the characteristics of the Wannier functions as discussed above. For $b>1.34 \mathrm{~nm}$, the trend is reversed: i.e., the smaller the $a$ the lower the plasmon energy.

Figure 14 displays the intersubband plasmon energy as a function of the well width for a given value of the momentum transfer $q / k_{F}=0.1$ and for several values of the barrier width. The discussion of this figure is very nearly the same as that of Fig. 12, except for the fact that here the 


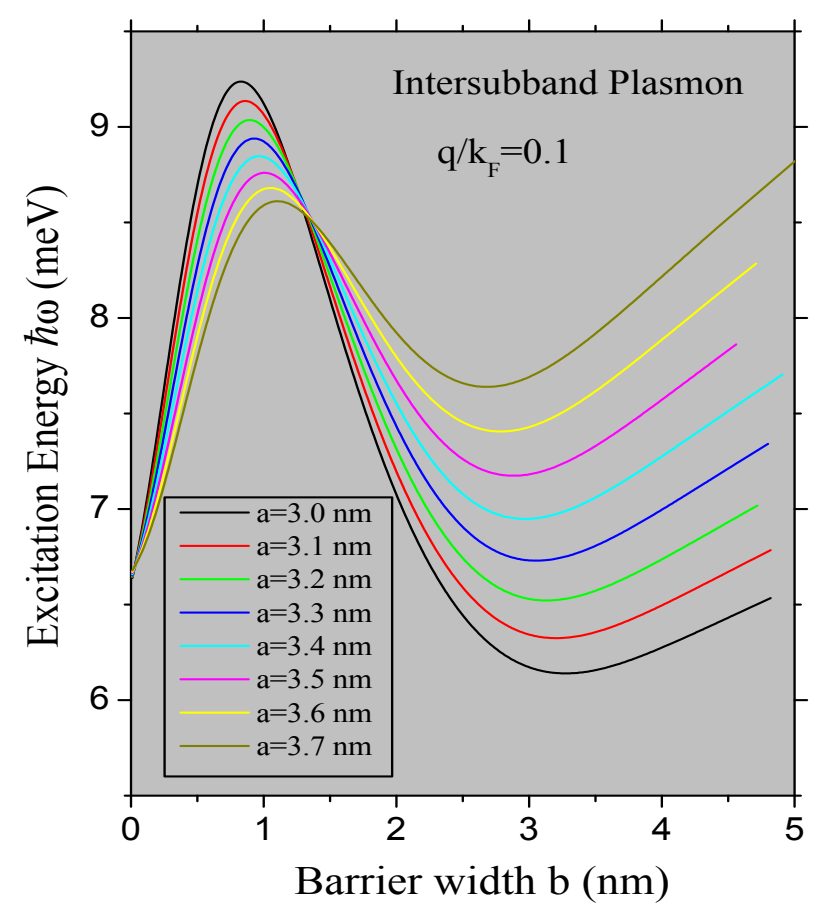

FIG. 13: (Color online) Intersubband plasmon dispersion vs. the barrier width for several values of the well width. The momentum transfer is fixed as $q / k_{F}=0.1$. The other parameters are the same as in Fig. 8.

focal point [where all the modes roughly cross each other] (at $a \simeq 4.0$ ) is rather sharper than it was in Fig. 12. We observe at a glance that the plasmon dispersion, in the range $0.0<a(\mathrm{~nm}) \lesssim 4.0$, obeys this rule: the lower the $b$, the higher the plasmon energy. This trend is, however, reversed after the focal point (i.e., for $a \gtrsim 4.0 \mathrm{~nm}$ ). Again, this is explicable in terms of the Wannier function and its behavior as a function of the well width.

\section{The dependence on the charge density}

Figure 15 shows the dependence of the collective (plasmon) excitations on the charge density of the resultant quantum wire system made up of the VSQD. It is noteworthy that we have plotted only the bonafide intrasubband and intersubband CPE which remain Landau-undamped until a very large propagation vector before merging with the respective SPE. The same-colored upper (lower) mode is the intersubband (intrasubband) plasmon designated as $\Omega_{10}\left(\Omega_{00}\right)$ in Fig. 8. Notice that the layer thicknesses are kept the same as in Fig. 8. In addition, we have purposely avoided to plot the respective single-particle continua in order not to make a mess in the picture. What we, generally, observe in this figure is what we intuitively expect: the energy of the plasmon 


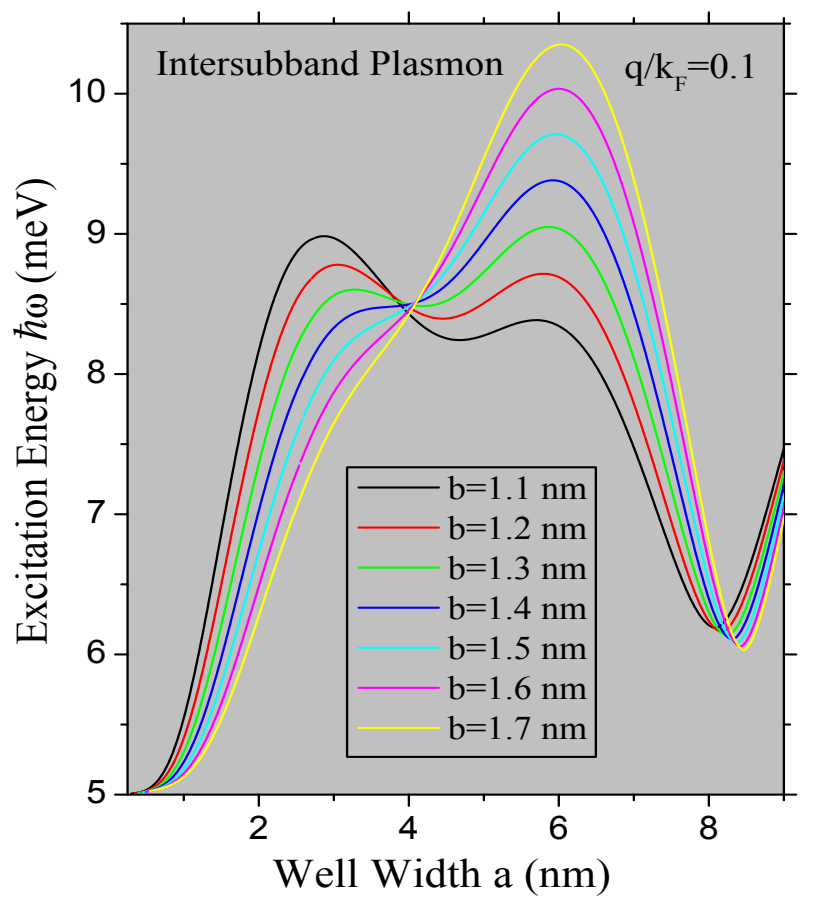

FIG. 14: (Color online) Intersubband plasmon dispersion vs. the well width for several values of the barrier width. The momentum transfer is fixed as $q / k_{F}=0.1$. The other parameters are the same as in Fig. 8.

excitations increases with increasing charge density. The literature is a live witness that this remark remains true irrespective of the size and dimension of the system and we are not aware of any exception to this rule. It is, however, interesting to note that the sharp dip in the intersubband $\mathrm{CPE}$ (where the group velocity changes the sign from negative to positive) is seen to be smoothed out with the lowering of the charge density. Another interesting effect the variation of the charge density can have on the excitation spectrum is that the single-particle continua (of intra- and inter-subband excitations) do not overlap for $n_{1 D} \lesssim 0.6 \times 10^{6} \mathrm{~cm}^{-1}$.

\section{On the inverse dielectric functions}

As mentioned before, the study of inverse dielectric function helps us substantiate the plasmon excitations generally searched through the zeros of the dielectric function. This is what we should anticipate because the zeros of the DF and the poles of the IDF must yield exactly identical results. There is an advantage of the latter over the former. A careful analysis of the IDF also reflects over the longitudinal (transverse or Hall) resistance in such quantum systems. For instance, the longitudinal (Hall) resistance $\rho_{z z}\left(\rho_{y z}\right)$ is determined by the imaginary (real) part of the IDF. In 


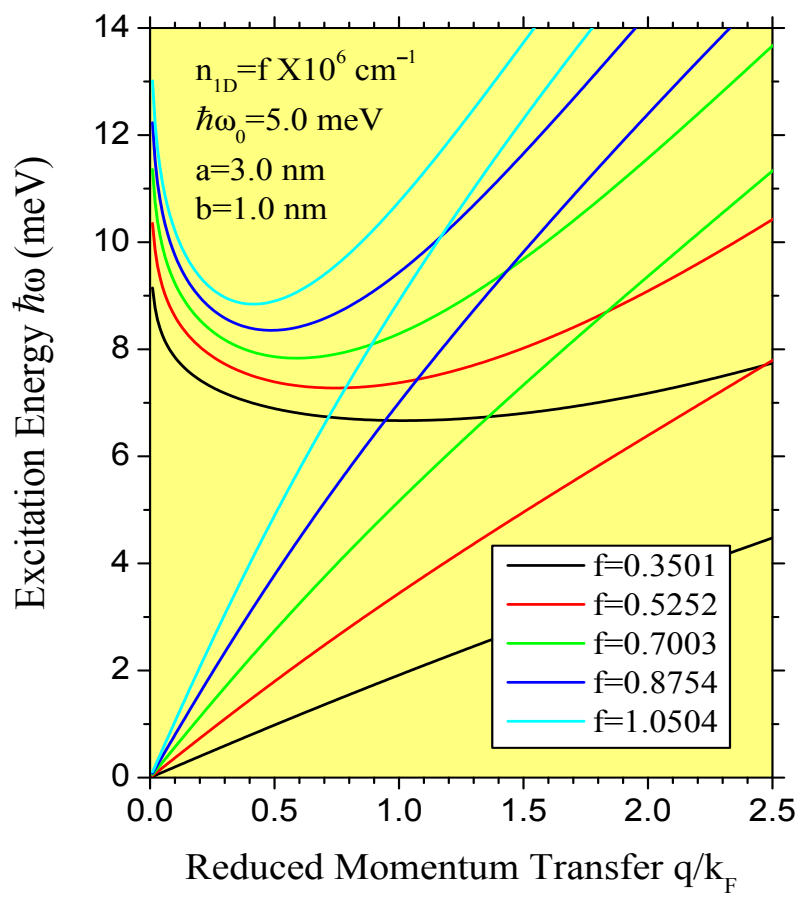

FIG. 15: (Color online) The collective (plasmon) excitation spectrum within a two-subband model where the energy $\hbar \omega$ is plotted as a function of the reduced momentum transfer $q / k_{F}$, for several values of the charge density $n_{1 D}$. The other parameters are as listed inside the picture. Notice that we have plotted only the bonafide intrasubband and intersubband plasmons which remain Landau-undamped until a very large propagation vector.

addition, the imaginary part of the IDF also furnishes very significant estimates of the Raman (or electron) scattering cross-section. In what follows, we will analyze the IDF computed in two cases: keeping the excitation energy (momentum transfer) fixed while varying the momentum transfer (excitation energy).

Figure 16 illustrates the $\operatorname{IDF} \epsilon^{-1}(q, \omega)$ as a function of the reduced momentum transfer $q / k_{F}$ for two given values of the excitation energy: $\hbar \omega=2.0 \mathrm{meV}$ and $9.0 \mathrm{meV}$. It is worth mentioning that the quantity that directly affects the transport phenomena is the spectral weight $\operatorname{Im}\left[\epsilon^{-1}(q, \omega)\right]$ which contains both the single-particle contribution at large momentum transfer $(q)$ and the collective (plasmon) contribution at small $q$. Looking at Fig. 8 reveals that the resonances corresponding to the excitation energy $\hbar \omega=2.0(9.0) \mathrm{meV}$ should yield the intrasubband (intersubband) excitations. This is exactly what we observe. To be explicit, for $\hbar \omega=2.0 \mathrm{meV}$, the sharp peak at $q / k_{F}=0.39$ produces the intrasubband collective (plasmon) mode while the crossing of the real and imaginary parts at $q / k_{F}=0.87$ yields the intrasubband single-particle excitation. The latter actually refers to the point on the upper edge of the intrasubband single- 


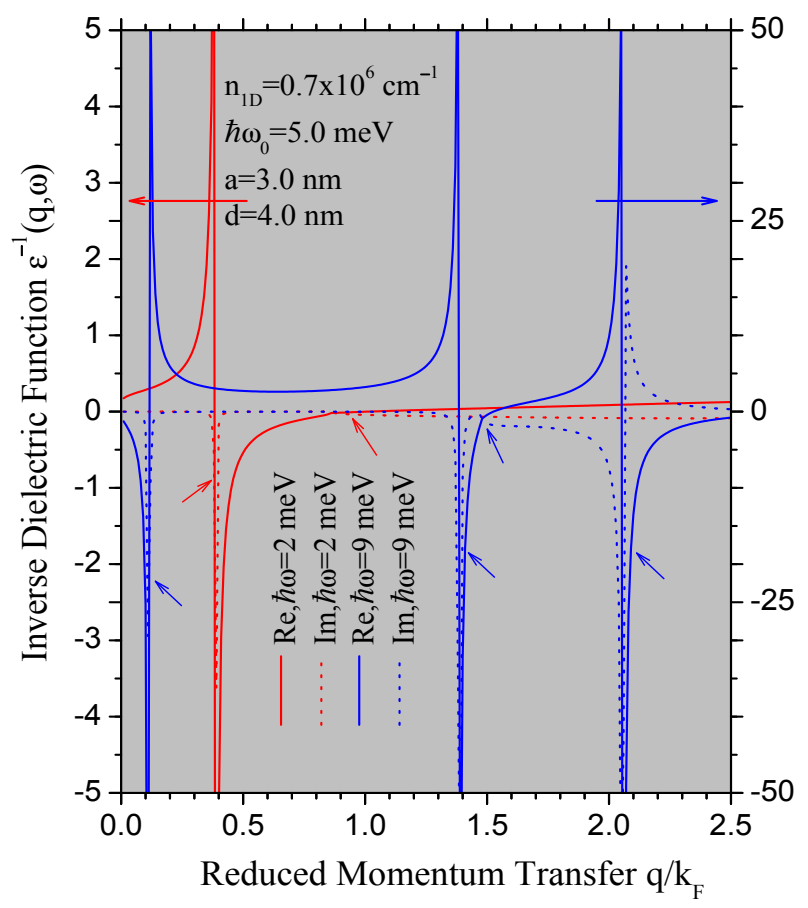

FIG. 16: (Color online) Inverse dielectric function $\epsilon^{-1}(q, \omega)$ vs. the momentum transfer $q / k_{F}$ for the given values of the excitation energy $\hbar \omega$. The other parameters are as listed inside the picture.

particle continuum. Similarly, for $\hbar \omega=9.0 \mathrm{meV}$, the lowest, second lowest, and third lowest sharp peaks (counting from the origin) at $q / k_{F}=0.11,1.39$, and 2.06 reproduce, respectively, the intersubband collective (plasmon) mode before the dip, after the dip, and the intrasubband plasmon within the intersubband single-particle continuum. The crossing of the real and imaginary parts at $q / k_{F}=1.48$ yields exactly the point on the upper edge of the intersubband single-particle continuum. Clearly, all these resonance peaks are a result of the existing poles of the IDF within the $\omega-q$ space.

Figure 17 shows the $\operatorname{IDF} \epsilon^{-1}(q, \omega)$ as a function of the excitation energy $\hbar \omega$ for the several values of the momentum transfer: $q / k_{F}=0.1,0.2,0.3,0.4$, and 0.5. Again, in analogy with the preceding discussion, we focus on the imaginary part of the IDF. Since we choose relatively small values of the momentum transfer, we should expect the resonance peaks to identify only the collective (plasmon) modes. We find two sharp peaks for a given momentum transfer where the lower (upper) peak yields the intrasubband (intersubband) collective plasmon. To be specific, the resonance peaks occurring at $\hbar \omega=0.53,1.06,1.57,2.07$, and 2.56 reproduce the intrasubband plasmon and those at $\hbar \omega=9.13,8.46,8.11,7.89$, and 7.77 yield the intersubband plasmon corresponding, respectively, to the momentum transfer $q / k_{F}=0.1,0.2,0.3,0.4$, and 0.5 . Notice how the higher resonance peaks are seen to exist in the reverse order to the values of $q$. This 


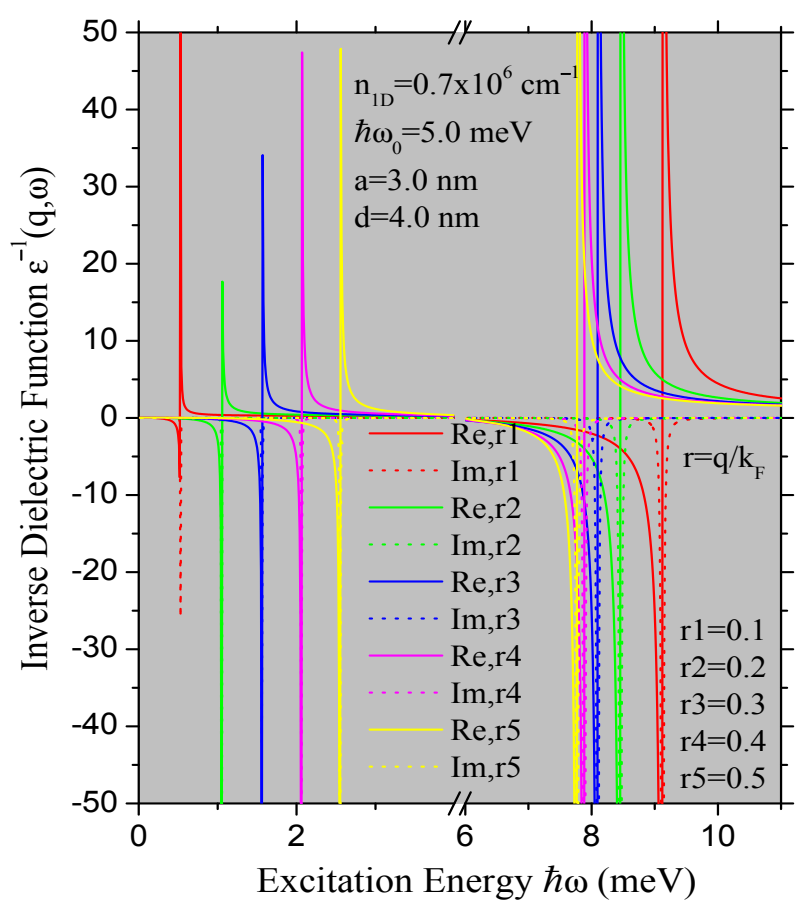

FIG. 17: (Color online) Inverse dielectric function $\epsilon^{-1}(q, \omega)$ vs. the excitation energy $\hbar \omega$ for the given values of the momentum transfer $q / k_{F}$. The other parameters are as listed inside the picture. Note the scale break on the abscissa.

is simply because the intersubband plasmon, for the chosen values of the $q$, propagates with the negative group velocity, until it observes the (roton-like) minimum. For the moment, we do not want to expand on the rotonic character of the intersubband collective excitation observed here and wish to leave it for the future.

\section{E. Comparison with the normal quantum wires}

A systematic investigation of the excitation spectrum - comprised of single-particle and collective excitations - in the quantum wires made up of vertically stacked quantum dots poses a question: how do we compare the coupled-dot quantum wires (CDQW) with the normal quantum wires (NQW). In order to answer this question, we look at the excitation spectra obtained in the two systems [see, e.g., Fig. 8 here and Fig. 133 in Ref. 1] and discuss some of the specific characteristics that distinguish the two systems apparently dubbed with the same nickname (i.e., quantum wires). These are, for instance, the following. (a) The energy scales are usually higher in the NQW than in the CDQW. (b) The intra- and inter-subband SPE (usually) overlap in the NQW unlike in the CDQW. (c) The minimum of the lower branch of the intersubband 
SPE, generally, approaches (very close to) zero in the NQW unlike in the CDQW. (d) Both intraand inter-subband CPE become Landau-damped at longer wavelengths in the NQW than in the CDQW. (e) The intersubband CPE, generally, starts to propagate with the positive group velocity in the NQW unlike in the CDQW. (f) The most important difference between the NQW and the CDQW is that the former offer us an (inherent) translational invariance (TI) whereas the latter are imposed upon such TI (due, in fact, to the smaller length scales in the problem) for the sake of (mathematical) convenience.

\section{CONCLUDING REMARKS}

In summary, we have investigated extensively the single-particle and collective (plasmon) excitations in the quantum wires made up of vertically stacked (self-assembled) quantum dots within a two-subband model in the framework of the Bohm-Pines' full RPA. The elementary characterization of such quantum wires by studying, e.g., the wave functions, miniband structure, band width, density of states, and Fermi energy is followed by the principal results on the single-particle and collective excitations, the influence of the well and barrier widths on the plasmon dispersion, and the inverse dielectric functions. As to the intricacy of the methodology, we would like to stress that although the TBA is, generally, labeled as the scheme accounting for the week tunneling effects, it is found to be reasonably well successful. This is attributed to the smaller length scales involved in the problem.

As to the similarities and differences between the homogeneous quantum wires and the quantum wires made up of the VSQD, we observe some important traits here. First of all, the presence of well and barrier widths provide us with the freedom to tailor the excitation spectrum in desired energy range. Secondly, both intrasubband and intersubband collective (plasmon) excitations are found to be free from Landau damping and comparatively long-lived in a greater range of propagation vector. Thirdly, the intersubband plasmon excitation is observed to be originating and propagating with a negative group velocity in the long wavelength limit. This is a roton character observed in the homogeneous quantum wires in the presence of an applied (perpendicular) magnetic field. We think this is an important issue and requires a separate extensive study. It is interesting to see how inseparable is the role played by the well and the barrier in influencing the plasmon propagation.

The motivation behind studying the inverse dielectric function is not solely to reaffirm the fact that the poles of the IDF and the zeros of the DF yield exactly identical excitation spectrum, but 
also to pinpoint the advantage of the former over the latter. For instance, the imaginary (real) part of the IDF sets to furnish a significant measure of the longitudinal (Hall) resistance in the system. Moreover, the quantity $\operatorname{Im}\left[\epsilon^{-1}(q, \omega)\right]$ also implicitly provides the reasonable estimates of the inelastic electron (or Raman) scattering cross-section $S(q)$ for a given system.

Finally, we believe that the present investigation of the plasmon excitations in a quantum wire made up of VSQD is of experimental importance given the excitement in the emerging fields of single-electron devices and the quantum computation. Considering the influence of an applied magnetic field, a confining potential that deviates from the parabolic form, and the many-body effects could give better insight into the problem. We hope that such behavior characteristics of the plasmons as studied and predicted here can be verified by the Raman scattering experiments.

\section{Acknowledgments}

During the course of this work the author has benefited from many stimulating discussions and communications with some colleagues. I would like to particularly thank B. Djafari-Rouhani, Naomi Halas, and Peter Nordlander. Special thanks are due to Peter Nordlander for critical reading of the manuscript. I also wish to acknowledge Kevin Singh for the generous help with the software throughout. It is a pleasure to thank Professor F. Barry Dunning for all the support and encouragement. 


\section{Appendix A: The relevance of the Kronig-Penney model}

Here, we want to capture briefly the essence of the classic Kronig-Penney (KP) model with the Bastard's boundary conditions (BBC) [44] incorporated. We do not want to pretend to be innovative since the KP model has so widely been treated in the textbooks. Our intent is twofold: the completeness of the eigenfunctions involved and to uncover an interesting consequence of applying the Bastard's boundary conditions. The KP model is represented by a 1D periodic potential shown in the right panel of Fig. 1. Even though the model is 1D, it is the periodicity of the potential that is crucial to the resulting electronic band structure. The periodic potential in the Schrödinger equation is defined such as

$$
V(z)= \begin{cases}V_{0}, & \text { if } \quad \mathrm{nd}+\mathrm{a}<\mathrm{z}<(\mathrm{n}+1) \mathrm{d} \\ 0, & \text { if } \quad \mathrm{nd}<\mathrm{z}<\mathrm{nd}+\mathrm{a}\end{cases}
$$

As shown in Fig. 1, the potential has the period $d=a+b$. The Schrödinger equation for this model is

$$
\begin{array}{r}
\frac{d^{2} u}{d z^{2}}+\frac{2 m_{w}^{*} \epsilon_{0}}{\hbar^{2}} u=0, \text { if } \mathrm{nd}<\mathrm{z}<\mathrm{nd}+\mathrm{a} \\
\frac{d^{2} u}{d z^{2}}-\frac{2 m_{b}^{*}\left(V_{0}-\epsilon_{0}\right)}{\hbar^{2}} u=0, \text { if } \mathrm{nd}+\mathrm{a}<\mathrm{z}<(\mathrm{n}+1) \mathrm{d}
\end{array}
$$

where $m_{w}^{*}\left(m_{b}^{*}\right)$ is the effective mass of the electron inside the well (barrier). The solutions to these equations are

$$
\begin{array}{r}
u_{1}(z)=A e^{i \alpha z}+B e^{-i \alpha z}, \text { if } \mathrm{nd}<\mathrm{z}<\mathrm{nd}+\mathrm{a} \\
u_{2}(z)=C e^{\beta(z-a)}+D e^{-\beta(z-a)}, \text { if } \quad \mathrm{nd}+\mathrm{a}<\mathrm{z}<(\mathrm{n}+1) \mathrm{d},
\end{array}
$$

where $\alpha=\sqrt{2 m_{w}^{*} \epsilon_{0} / \hbar^{2}}$ and $\beta=\sqrt{2 m_{b}^{*}\left(V_{0}-\epsilon_{0}\right) / \hbar^{2}}$ are real quantities if we assume for the time being that $\epsilon_{0}<V_{0}$. Next, we need to match the boundary conditions at $z=a$ and $z=d$ along with the Bloch theorem. These boundary conditions are:

$$
\begin{aligned}
u_{1}(a) & =u_{2}(a) \\
\frac{1}{m_{w}^{*}} u_{1}^{\prime}(a) & =\frac{1}{m_{b}^{*}} u_{2}^{\prime}(a) \\
u_{1}(0) & =e^{i k d} u_{2}(d) \\
\frac{1}{m_{w}^{*}} u_{1}^{\prime}(0) & =\frac{1}{m_{b}^{*}} e^{i k d} u_{2}^{\prime}(d)
\end{aligned}
$$


Eqs. (A7) and (A9) are becoming known as the BBC. A careful matching of these boundary conditions yields:

$$
\begin{aligned}
A e^{i \alpha a}+B e^{-i \alpha a} & =C+D \\
A e^{i \alpha a}-B e^{-i \alpha a} & =S(C+D) \\
A+B & =e^{i k d}\left[C e^{\beta b}+D e^{-\beta b}\right] \\
A-B & =S e^{i k d}\left[C e^{\beta b}-D e^{-\beta b}\right],
\end{aligned}
$$

where $k$ is the Bloch vector and $S=-i\left(\beta / m_{b}^{*}\right) /\left(\alpha / m_{w}^{*}\right)$. In order for there to be a nontrivial solution to the Eqs. $(A 10)-(A 13)$ for $A, B, C$, and $D$, the determinant of their coefficients must vanish. This yields the determinantal equation

$$
\left|\begin{array}{cccc}
e^{i \alpha a} & e^{-i \alpha a} & -1 & -1 \\
e^{i \alpha a} & -e^{-i \alpha a} & -S & S \\
1 & 1 & -e^{i k d} e^{\beta b} & -e^{i k d} e^{-\beta b} \\
1 & -1 & -S e^{i k d} e^{\beta b} & S e^{i k d} e^{-\beta b}
\end{array}\right|=0
$$

It is straightforward, albeit lengthy, to simplify Eq. (A14) and prove that it is equivalent to

$$
\cos (k d)=\cos (\alpha a) \cosh (\beta b)+i \frac{1+S^{2}}{2 S} \sin (\alpha a) \sinh (\beta b)
$$

for $\beta$ real and

$$
\cos (k d)=\cos (\alpha a) \cos (\gamma b)-\frac{1+S^{2}}{2 S} \sin (\alpha a) \sin (\gamma b)
$$

for $\beta(=i \gamma)$ imaginary. This is the classic (electron) dispersion relation for the 1D periodic system in the KP model, but with the BBC embodied. For $m_{w}^{*}=m^{*}=m_{b}^{*}$, it exactly simplifies to the textbook result. Analytically, Eq. (A15) may appear to be somewhat cumbersome, but it may be cast in a more transparent form. Let $\alpha a=c_{1} \sqrt{\epsilon}$ and $\beta b=c_{2} \sqrt{1-\epsilon}$, where $\epsilon=\epsilon_{0} / V_{0}, c_{2}=r c_{1}$, $c_{1}=\sqrt{2 m_{w}^{*} V_{0} / \hbar^{2}} a, r=r_{l} \sqrt{r_{m}}, r_{l}=b / a$, and $r_{m}=m_{b}^{*} / m_{w}^{*}$. Then, Eq. (15) can be written in the form

$$
\cos (k d)=\cos \left(c_{1} \sqrt{\epsilon}\right) \cosh \left(c_{2} \sqrt{1-\epsilon}\right)+\frac{1-\epsilon\left(1+r_{m}\right)}{2 \sqrt{r_{m}} \sqrt{\epsilon} \sqrt{1-\epsilon}} \sin \left(c_{1} \sqrt{\epsilon}\right) \sinh \left(c_{2} \sqrt{1-\epsilon}\right)
$$

for $\epsilon<1$ and

$$
\cos (k d)=\cos \left(c_{1} \sqrt{\epsilon}\right) \cos \left(c_{2} \sqrt{\epsilon-1}\right)+\frac{1-\epsilon\left(1+r_{m}\right)}{2 \sqrt{r_{m}} \sqrt{\epsilon} \sqrt{\epsilon-1}} \sin \left(c_{1} \sqrt{\epsilon}\right) \sin \left(c_{2} \sqrt{\epsilon-1}\right)
$$

for $\epsilon>1$. In the limit of $\epsilon \rightarrow 1$, both Eqs. (16) and (17) yield, with $r_{t}=r_{l} r_{m}$,

$$
\cos (k d)=\cos \left(c_{1}\right)-\frac{r_{t} c_{1}}{2} \sin \left(c_{1}\right)
$$


It is instructive to subject Eq. (15) to the limit of a homogeneous medium. This does not simply mean only to put $V_{0}=0$ but also to take $r_{m}=1$ in Eq. (15). In that case $\beta=i \alpha$ and Eq. (15) yields $\alpha=k$. Therefore, we find $\epsilon_{0}=\hbar^{2} k^{2} / 2 m^{*}$ for a free electron.

Next, we need to determine fully the wave functions in Eqs. $(A 4)-(A 5)$. For this purpose, we first write the coefficients $B, C$, and $D$ in terms of $A$ from Eqs. $(A 10)-(A 13)$ and then apply the condition of normalization

$$
\frac{N}{2}\left\{\int_{0}^{a} d z u_{1}^{*}(z) u_{1}(z)+\int_{a}^{d} d z u_{2}^{*}(z) u_{2}(z)\right\}=1,
$$

in order to determine $A$. Thus, the four coefficients $A, B, C$, and $D$ are defined as follows.

$$
\begin{aligned}
& A=\frac{1}{\sqrt{N}}\left[a \frac{\cos (\alpha a) \cos (k d)-\cosh (\beta b)}{\cos (\alpha a-k d)-\cosh (\beta b)}+\frac{b}{t} \frac{\sin (\alpha a)}{\sinh (\beta b)} \frac{\cos (\alpha a)-\cos (k d) \cosh (\beta b)}{\cos (\alpha a-k d)-\cosh (\beta b)}\right]^{1 / 2} \\
& B=-R^{-1}\left(1-S^{2}\right) \sinh (\beta b) A \\
& C=r^{-1} R^{-1}(1+S) e^{-\beta b}\left[r e^{-i \alpha a}-\cosh (\beta b)-\sinh (\beta b)\right] A \\
& D=-r^{-1} R^{-1}(1-S) e^{\beta b}\left[r e^{-i \alpha a}-\cosh (\beta b)+\sinh (\beta b)\right] A,
\end{aligned}
$$

where $t=i S, r=e^{i k d}$, and $R=2 S\left[r e^{-i \alpha a}-\cosh (\beta b)\right]-\left(1+S^{2}\right) \sinh (\beta b)$. Thus the wave functions $u_{1}(z)$ and $u_{2}(z)$ in Eqs. $(A 4)-(A 5)$ become finally known. The coefficient $A$ subjected to the limit of a homogeneous medium and then substituting $k=0$ yields $A=1 / \sqrt{N d}$, just as expected. A word of warning: you may never obtain the correct result if you reverse the order of the procedure, i.e. if you substitute $k=0$ first and then apply the condition of homogeneity (i.e., $\beta=i \alpha$ ). 
1 For an extensive review of electronic, optical, and transport phenomena in the systems of reduced dimensions, such as quantum wells, wires, dots, and electrically/magnetically modulated 2D systems, see M.S. Kushwaha, Surf. Sci. Rep. 41, 1 (2001).

2 H. Sakaki, Jpn. J. Appl. Phys. 28, L314 (1989).

3 Q. Xie, A. Madhukar, P. Chen, and N.P. Kobayashi, Phys. Rev. Lett. 75, 2542 (1995).

4 G.S. Solomon, J.A. Trezza, A.F. Marshall, and J.S. Harris, Phys. Rev. Lett. 76, 952 (1996).

5 M.S. Miller, Jpn. J. Appl. Phys. 36, 4123 (1997).

6 D.G. Austing, S. Sasaki, S. Tarucha, S.M. Reimann, M. Koskonen, and M. Manninen, Phys. Rev. B 60, 11514 (1999).

7 M. Bayer, P. Hawrylak, K. Hinzer, S. Fafard, M. Korkusinski, Z.R. Wasilewski, O. Stern, and A. Forchel, Science 291, 451 (2001).

8 I. Shtrichman, C. Metzner, B.D. Gerardot, W.V. Schoenfeld, and P.M. Petroff, Phys. Rev. B 65, 081303 (2002).

9 T. Maltezopoulos, A. Bolz, C. Meyer, C. Heyn, W. Hansen, M. Morgenstern, and R. Wiesendanger, Phys. Rev. Lett. 91, 196804 (2003).

10 T. Ota, K. Ono, M. Stopa, T. Hatano, S. Tarucha, H.Z. Song, Y. Nakata, T. Miyazawa, T. Oshima, and N. Yokoyama, Phys. Rev. Lett. 93, 066801 (2004).

11 T. Ota, M. Rontani, S. Tarucha, Y. Nakata, H.Z. Song, T. Miyazawa, T. Usuki, M. Takatsu, and N. Yokoyama, Phys. Rev. Lett. 95, 236801 (2005).

12 W.J.M. Naber, T. Fujisawa, H.W. Liu, and W.G. van der Wiel, Phys. Rev. Lett. 96, 136807 (2006).

13 H.J. Krenner, E.C. Clark, T. Nakoaka, M. Bichler, C. Scheurer, G. Abstreiter, and J.J. Finley, Phys. Rev. Lett. 97, 076403 (2006).

14 F.H. L. Koppens, C. Buizert, K. J. Tielrooij, I.T. Vink, K.C. Nowack, T. Meunier, L.P. Kouwenhoven, and L.M.K. Vandersypen, Nature 442, 766 (2006).

15 M.F. Doty, M. Scheibner, I.V. Ponomarev, E.A. Stinaff, A.S. Bracker, V.L. Korenev, T.L. Reinecke, and D. Gammon, Phys. Rev. Lett. 97, 197202 (2006).

16 K.C. Nowack, F.H.L. Koppens, Yu.V. Nazarov, and L.M.K. Vandersypen, Science 318, 1430 (2007).

17 T. Hatano, S. Amaha, T Kubo, Y. Tokura, Y. Nishi, Y. Hirayama, and S. Tarucha, Phys. Rev. B 77, 241301(R) (2008).

18 M.F. Doty, M. Scheibner, A.S. Bracker, I.V. Ponomarev, T.L. Reinecke, and D. Gammon, Phys. Rev. 
B 78, 115316 (2008).

19 S. Fält, M. Atatüre, H.E. Türeci, Y. Zhao, A. Badolato, and A. Imamoglu, Phys. Rev. Lett. 100, 106401 (2008).

20 T. Kodera, K. Ono, Y. Kitamura, Y. Tokura, Y. Arakawa, and S. Tarucha, Phys. Rev. Lett. 102, $146802(2009)$.

21 M.F. Doty, J.I. Climente, A. Greilich, M. Yakes, A.S. Bracker, and D. Gammon, Phys. Rev. B 81, $035308(2010)$.

22 C. Payette, S. Amaha, G. Yu, J.A. Gupta, D.G. Austing, S.V. Nair, B. Partoens, and S. Tarucha, Phys. Rev. B 81, 245310 (2010).

23 J.A. Fan, C. Wu, K. Bao, J. Bao, R. Bardhan, N.J. Halas, V.N. Manoharan, P. Nordlander, G. Shvets, and F. Capasso, Science 328, 1135 (2010).

24 M.A. Cusack, P.R. Briddon, and M. Jaros, Phys. Rev. B 54, R2300 (1996).

25 B. Partoens, V.A. Schweigert, and F.M. Peeters, Phys. Rev. Lett. 79, 3990 (1997).

26 C. Pryor, Phys. Rev. Lett. 80, 3579 (1998).

27 B. Partoens, A. Matulis, and F.M. Peeters, Phys. Rev. B 59, 1617 (1999).

28 C. Steinebach, C. Schuller, and D. Heitmann, Phys. Rev. B 61, 15600 (2000).

29 M. Meixner and E. Schöll, Phys. Rev. B 67, 121202(R) (2003).

30 G. Bester, J. Shumway, and A. Zunger, Phys. Rev. Lett. 93, 047401 (2004).

31 D. Bellucci, M. Rontani, F. Troiani, G. Goldoni, and E. Molinari, Phys. Rev. B 69, 201308(R) (2004).

32 J.I. Climente and J. Planelles, Phys. Rev. B 72, 155322 (2005).

33 W. Jaskolski, M. Zielinski, G.W. Bryant, and J. Aizpurua, Phys. Rev. B 74, 195339 (2006).

34 T.T. Kang, R.Q. Zhang, W.G. Hu, G.W. Kong, F.A. Zhao, X.X. Han, S.Y. Yang, X.L. Liu, Q.S. Zhu, and Z.G. Wang, Phys. Rev. B 76, 075345 (2007). This reference came to our attention after (almost) completion of the present work. These authors used the effective-mass approximation (EMA) and studied only the intrasubband plasmon in an extremely long wavelength limit. While their conclusions are a part of our detailed investigation, the EMA seems to have led them, in general, to overestimate the plasmon energies. Nevertheless, future experiments on the plasmon observation in such quantum wires as made up of VSQD should be able to clarify the discrepancy.

35 Y.Y. Wang and M.W. Wu, Phys. Rev. B 77, 125323 (2008).

36 S. Tomic, T.S. Jones, and N.M. Harrison, Appl. Phys. Lett. 93, 263105 (2008).

37 T. Andlauer and P. Vogl, Phys. Rev. B 79, 045307 (2009).

38 W. Sheng and J. Wang, Phys. Rev. B 82, 073308 (2010). 
39 H.Y. Ramirez and S.J. Cheng, Phys. Rev. Lett. 104, 206402 (2010).

40 J. Planelles, J.I. Climente, F. Rajadell, M.F. Doty, A.S. Bracker and D. Gammon, Phys. Rev. B 82, 155307 (2010).

41 D. Pines and P. Nozieres, The Theory of Quantum Liquids (Benjamin, New York, 1966); A.L. Fetter and J.D. Walecka, Quantum Theory of Many-Particle Systems (McGraw-Hill, New York, 1971); G.D. Mahan, Many Particle Physics (Plenum, New York, 1981).

42 B.Y.K. Hu and S. Das Sarma, Phys. Rev. Lett. 68, 1750 (1992); Phys. Rev. B 48, 5469 (1993).

43 M.S. Kushwaha and F. Garcia-Moliner, Phys. Lett. A 205, 217 (1995).

44 G. Bastard, Phys. Rev. B 24, 5693 (1981). The so-called "Bastard's boundary conditions" were known in the literature much before Bastard used them. See, e.g., D.J. BenDaniel and C.B. Duke, Phys. Rev. 152, 683 (1966).

45 H.B. Dwight, Tables of Integrals and Other Mathematical Data (Macmillan, New York, 1947); I.S. Gradshteyn and I.M. Ryzhik, Tables of Integrals, Series, and Products (Academic, New York, 1994); Wolfram Research, Inc., Mathematica, Version 7.0 (Champaign, IL, 2008). 